\title{
NUMERICAL PARAMETRIC STUDY ON INTERVAL SHIFT VARIATION IN SIMO-SSTD TECHNIQUE FOR EXPERIMENTAL MODAL ANALYSIS (EMA)
}

\author{
Ahmad Fathi Ghazali ${ }^{1}$, Ahmad Azlan Mat Isa ${ }^{1}$ \\ ${ }^{I}$ Faculty of Mechanical Engineering, Universiti Teknologi MARA, 40450 Shah Alam,Selangor D.E., Malaysia.
}

\begin{abstract}
SSTD (Single Station Time Domain) technique is one of the efficient methods used to extract modal parameters of a structure. Instead of extracting the parameters in frequency domain by utilising Fast Fourier Transform (FFT) as used by some other methods, this technique only relies on free decay responses to obtain the required parameters. In this paper, a parametric study on time shift interval on algorithm method is investigated. The accuracy assessment is made using simulated analytical data with known properties where percentage error between the results of simulated and this method can be calculated and compared.
\end{abstract}

Keywords: SSTD (Single Station Time Domain), FFT, EMA

\section{INTRODUCTION}

One objective of doing modal analysis is to extract dynamic characteristics of a system in terms of natural frequencies, mode shapes and damping ratios. They are normally known as modal parameters. The knowledge of these parameters can feed various purposes including structural modification and health monitoring, model updating, assessment of the structural integrity and reliability to name a few [1, 2]. A number of literature reported that the applications cover wide range of engineering, science and technology fields namely in aerospace, automotive, mechanical, civil, musical instruments, medicine as well as bioengineering for example [3-5].

One way of determining modal parameters is via theoretical modal analysis which assumes the information of stiffness matrix $\mathrm{K}$, mass matrix $\mathrm{M}$ and damping matrix $\mathrm{C}$ to be known. However, it is usually difficult to have a reliable mathematical model that can accurately represent dynamic behaviour of different components and of the complete assembly. This include difficulties not only in modelling joints, welding and other type of connections but also in describing damping in an adequate manner [6].

Second approach is by experimental, popularly known as 'experimental modal analysis (EMA)'. Due to inadequacy of analytical modelling of structures, endeavours on experimental or combined experimental method have become centre of attention as seen by the diversity developments of modal parameter identification techniques since 1970's. With the revolutionary advent of Fast Fourier Transform (FFT) by Cooley and Tukey at that time as well, EMA has grown steadily and become widespread [7]. The techniques can be classified into two categories; frequency and time domain.

The general idea behind the frequency domain methods is a curve fitting process done on a set of function known as
Frequency Response Function (FRF). This function is simply a ratio between the output responses to the input excitation force. In practise, this is obtained by transforming the raw time signal data to FRF using FFT algorithm found in any signal processing analyser or in computer software packages before it is curve fitted. Curve fitting is basically a process of matching a predefined mathematical expression to the measured data. Thus, the matching objective is to minimize the error function defined between the analytical expression and measured data before any process of extracting modal parameters can be made. Clearly, the accuracy will depend on the quality of measured FRF as well as correct mathematical model used. Examples of this identifications are Rational Fractional Polynomial (RFP) [811], Ewins-Gleeson method [12] and Simultaneous Frequency Domain (SFD) [3] .

A number of drawbacks have been reported such as aliasing, leakage, requirement for large amount of data, sampling and quantization error during measurement. Most of these shortcomings were aided with the expansion of technology in instruments precision, fast processing hardware, storage capacity device as well as advancement in measurement techniques. This is true except for leakage error. So far this is the greatest challenge for frequency domain methods as the error cannot be eliminated. Briefly, the Fourier Transform process demanded sampled data consist of complete representation of data for all time or contain periodic repetition of the measured data. When this is not the case as this is what exactly happen in practice, leakage occur causing serious distortion of data. Although windowing is applied to reduce the problem, the technique could give harmful side effect on the accuracy of modal parameter estimation especially damping [13, 14]. Other problems associated with frequency domain methods are listed in Ref [15].

Due to the aforementioned issues, the inertia of research tends to shift back to the use of time domain. There are 
several advantages using this domain. Unlike FRF, time domain methods manipulate only time signal of response instead the need to measure excitation force. Thus, less information is needed and no issue of leakage will take place. It is also reported that the methods are able to differentiate closed mode which has been a problem among frequency domain methods [16].

However, this type of methods has its own challenges. This includes high sensitivity towards noise and the 'invisibility' of structural modes in the time signal. The existence of noise may pave to computational modes in the calculation which they are not the genuine vibration mode but nevertheless 'identified'. Some examples of this type technique are Ibrahim Time Domain (ITD) [16-18], Single Station Time Domain (SSTD) [19], Complex Exponential (CE) and Eigensystem Realization Algorithm (ERA).

\section{THEORETICAL BACKGROUND}

This section outlined the SSTD algorithm including parts where different shift intervals are used in the study. More detailed explanation regarding the algorithm can be referred to the paper written by Zaghlool [19].

\subsection{SSTD Algorithm}

SSTD method is a variant of Ibrahim Time Domain (ITD) method as both techniques share the same way on how the algorithm works. The difference is that ITD is a SingleInput-Multiple-Output (SIMO) type while SSTD is a SingleInput-Single-Output (SISO) type [16]. In order to make SSTD as a SIMO type, the algorithm will include least square method as explained by Mohanty [20].

Considering at time $t_{j}$, a system with $N$ structural modes can be expressed as a summation of the individual responses specific at point $i$ and time $j$ of each mode $r$ :

$$
x_{i}\left(t_{j}\right)=\sum_{r=1}^{2 N} p_{i r} e^{s_{r} t_{j}}
$$

where $p_{i r}$ is the eigenvector at point $i$ for mode $r$. In this algorithm, $N$ refers to the number of identification order which will identify converged modal parameters value instead of defining $N$ as number of structural modes. In addition, the total number of converged modal parameters will be the number of structural mode for that system. From (1), the response measured at $L$ instances of time can be written as follow:

$$
\underset{2 N \times L}{[\boldsymbol{X}]}=\underset{2 N \times 2 N}{[\boldsymbol{P}]} \underset{2 N \times L}{[\boldsymbol{\Lambda}}]
$$

Another set of equation that is exactly similar to (2) but is shifted by $\Delta t_{l}$ is expressed as below

$$
\begin{aligned}
& x_{i}\left(t_{j}+\Delta t_{1}\right)=\sum_{r=1}^{2 N} p_{i r} e^{s_{r}\left(t_{j}+\Delta t_{1}\right)} \\
& =\sum_{r=1}^{2 N} \hat{p}_{i r} e^{s_{r} t_{j}} \\
& \hat{p}_{i r}=p_{i r} e^{s_{r} \Delta t_{1}}
\end{aligned}
$$

This can also be written in symbolic form as below:

$$
\underset{2 N \times L}{[\hat{\boldsymbol{X}}]}=\underset{2 N \times 2 N}{[\hat{\boldsymbol{P}}]} \underset{2 N \times L}{[\boldsymbol{\Lambda}]}
$$

A square matrix $\left[A_{s}\right]$ of order $2 N$ is defined as below:

$$
\underset{2 N \times 2 N}{[\hat{\boldsymbol{P}}]}=\underset{2 N \times 2 N}{\left[\boldsymbol{A}_{\boldsymbol{s}}\right][\boldsymbol{P}]}
$$

Multiply (2) by $\left[A_{s}\right]$ yield

$$
\underset{2 N \times 2 N 2 N \times L}{\left[\boldsymbol{A}_{s}\right][\boldsymbol{X}]}=\underset{2 N \times 2 N}{\left.\left[\boldsymbol{A}_{s}\right] \underset{2 N \times 2 N}{[\boldsymbol{P}}\right][\boldsymbol{\Lambda}]}
$$

Substitute (6) into (7) leads to

$$
\underset{2 N \times 2 N}{\left[\boldsymbol{A}_{s}\right]}[\underset{2 N \times L}{\boldsymbol{X}}]=\underset{2 N \times 2 N}{[\hat{\boldsymbol{P}}]} \underset{2 N \times L}{[\boldsymbol{\Lambda}]}
$$

Insert (5) into (8) gives

$$
\left.\left.\underset{2 N \times 2 N}{\left[\boldsymbol{A}_{s}\right]} \underset{2 N \times L}{[\boldsymbol{X}}\right]=\underset{2 N \times L}{[\hat{\boldsymbol{X}}}\right]
$$

As stated earlier, Equation (9) use a single output signal with respect to a single input. Hence, a least square approach is used in order to get a SIMO-SSTD type as Matrix $\left[\mathrm{A}_{\mathrm{s}}\right]$ is independent of the location of measurements and remain valid for any SISO combination [20]. Transforming Equation (9) for $n$ responses due to single input can be outlined as below:

$$
\left.\underset{2 N \times 2 N}{\left[\boldsymbol{A}_{s}\right.}\right]\left[\begin{array}{cccc}
\boldsymbol{X}_{1} & \boldsymbol{X}_{2} & \cdots & \boldsymbol{X}_{n} \\
2 N \times L & \substack{2 N \times L \\
2 N \times n L}
\end{array}\right]=\left[\begin{array}{cccc}
\hat{\boldsymbol{X}}_{1} & \hat{\boldsymbol{X}}_{2} & \cdots & \hat{\boldsymbol{X}}_{\boldsymbol{n}} \\
2 N \times L & 2 N \times L \\
2 N \times L & & 2 N \times L
\end{array}\right]
$$

where $\left[\boldsymbol{X}_{\boldsymbol{n}}\right]$ refer to matrix with point $i$ and time $j$ as explained in (1). In more compact form,

$$
\underset{2 N \times 2 N}{\left[\boldsymbol{A}_{s}\right]} \underset{2 N \times n L}{[\boldsymbol{Y}]}=\underset{2 N \times n L}{[\hat{\boldsymbol{Y}}]}
$$

In order to find Matrix $\left[\mathrm{A}_{\mathrm{s}}\right]$, pseudo inverse technique is used to give an expression known as Double Least-Squares 
(DLS). It is claimed that the equation below result better estimates of the damping factors [21].

$$
\left[\boldsymbol{A}_{s}\right]=\frac{1}{2}\left[\left([\hat{\boldsymbol{Y}}][\hat{\boldsymbol{Y}}]^{T}\right)\left([\boldsymbol{Y}][\hat{\boldsymbol{Y}}]^{T}\right)^{-1}+\left([\hat{\boldsymbol{Y}}][\boldsymbol{Y}]^{T}\right)\left([\boldsymbol{Y}][\boldsymbol{Y}]^{T}\right)^{-1}\right]
$$

From (6), the eigenvalues are deduced from matrix $\left[\mathrm{A}_{\mathrm{s}}\right]$ as below:

$$
\underset{2 N \times 2 N}{\left[\boldsymbol{A}_{s}\right]}\left\{\begin{array}{c}
p_{r} e^{s_{r} 0 \Delta t_{1}} \\
p_{r} e^{s_{r} 1 \Delta t_{1}} \\
\vdots \\
p_{r} e^{s_{r}(2 N-1) \Delta t_{1}}
\end{array}\right\}=\left\{\begin{array}{c}
p_{r} e^{s_{r} 0 \Delta t_{1}} \\
p_{r} e^{s_{r} 1 \Delta t_{1}} \\
\vdots \\
p_{r} e^{s_{r}(2 N-1) \Delta t_{1}} \\
2 N \times 1
\end{array}\right\} e^{s_{r} \Delta t_{1}} \quad r=1,2 \ldots 2 N
$$

Rearranging (13) to have a standard eigenvalue problem as follow:

$$
\left[\boldsymbol{A}_{s}-e^{s_{r} \Delta t_{1}} \boldsymbol{I}\right]\left\{\begin{array}{c}
p_{r} e^{s_{r} O \Delta t_{1}} \\
p_{r} e^{s_{r} 1 \Delta t_{1}} \\
\vdots \\
p_{r} e^{s_{r}(2 N-1) \Delta t_{1}}
\end{array}\right\}=\mathbf{O}
$$

For underdamped case:

$$
\begin{aligned}
s_{r} & =a_{r} \pm i b_{r} \\
& =-\xi_{r} \omega_{r} \pm i \omega_{r} \sqrt{1-\xi_{r}^{2}}
\end{aligned}
$$

The relationship between the eigenvalues (which is in complex form, $\beta_{r}+i \gamma_{r}$ ) and natural frequency are as follow:

$$
\begin{aligned}
f_{r} & =\frac{\left|\ln \left(\beta_{r}+i \gamma_{r}\right)\right|}{2 \pi \Delta t} \\
& =\frac{\left|a_{r}+i b_{r}\right|}{2 \pi}
\end{aligned}
$$

For damping ratio:

$$
\begin{gathered}
a_{r}=-\xi_{r} \omega_{r} \\
\xi_{r}=\frac{-a_{r}}{\omega_{r}}
\end{gathered}
$$

Modal Confidence Factor (MCF) is then applied to differentiate between genuine structural modes and computational modes for each $N$. If $p_{i j}$ is the $i^{\text {th }}$ element of the $j^{\text {th }}$ identified eigenvector or $j^{\text {th }}$ mode at the actual measurement, then the same measurement delayed at $\Delta t_{3}=10 \Delta t_{1}$ is expected to be:

$$
\overline{p_{i j}}=e^{\lambda_{j} \Delta t_{3}} p_{i j}
$$

if this identified eigenvector is a structural mode [16, 22]. Thus, MCF can be defined as the ratio between left and right hand side of (18) as below:

$$
\begin{aligned}
M C F_{i j} & =\left|\frac{e^{\lambda_{j} \Delta t_{3}} p_{i j}}{\overline{p_{i j}}}\right| \text { for } \overline{p_{i j}}>e^{\lambda_{j} \Delta t_{3}} p_{i j} \\
& =\left|\frac{\overline{p_{i j}}}{e^{\lambda_{j} \Delta t_{3}} p_{i j}}\right| \text { for } e^{\lambda_{j} \Delta t_{3}} p_{i j}>\overline{p_{i j}}
\end{aligned}
$$

and should be near unity for genuine structural mode. The selected measurements from MCF filter are then listed and grouped. Average measurements for each group will be calculated to obtain the final result for natural frequency and damping ratio.

\subsection{Modified SSTD Algorithm}

The idea of both SSTD and ITD methods is to reorder all the output or time signal responses to become a standard eigenvalue problem and solve it to get the dynamics characteristics. One of the parameter involve in the process is the shift interval. Mathematically they are described from equation (2) and (5). As the objective of this paper is to observe the impact of varying different shift interval towards the accuracy of natural frequency and damping ratio, following cases are considered for both matrices $[\mathbf{X}]$ and $\left[\mathbf{X}^{\wedge}\right]$ where the time interval is shifted with:

1) multiple by 1 (default settings in the algorithm)

2) multiple by 2

3) multiple by 3

4) multiple by 4

5) multiple by 5

\section{ANALYSIS AND PROCEDURES}

Matlab was used in the analysis where five simulated data with known properties were generated. Unlike previous work, these five simulated data will be tested in six different situations corrupted with distinct value of 'white Gaussian noise'. In other words, there were six different situations where each situation consists of five simulated data sets. The reason for using five simulated data for each situation is to simulate the response of a system at five various locations when an input was subjected at one of these points. An 


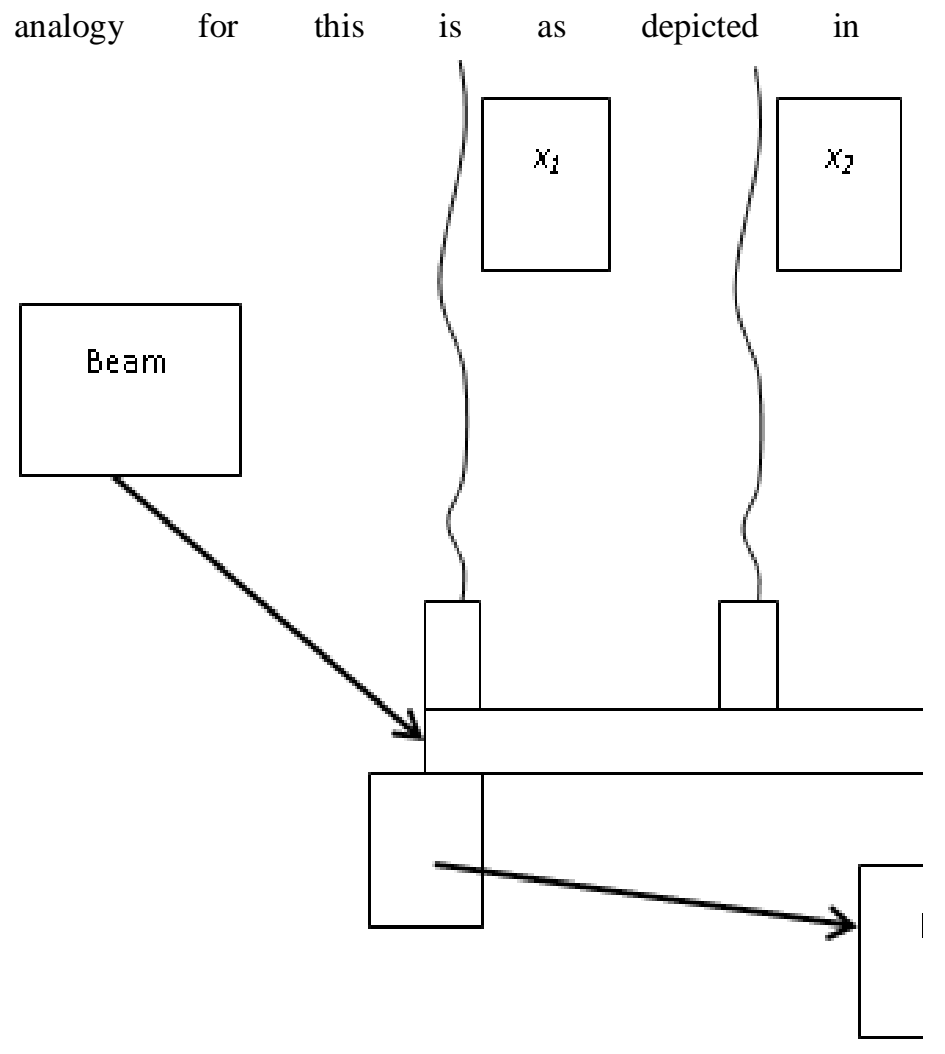

Fig 1 where a beam with five accelerometers at these points is subjected with an input at each point separately. When an input applied at one point results five responses for each points, this means that there are 25 responses when the input is subjected at all points. It is set up that for each data set to have five degree of freedom. The following table summarizes the details of the simulated data:

Table 1: Data collection parameters

\begin{tabular}{|l|l|}
\hline Parameter & Value \\
\hline Sample rate & $2048 \mathrm{~Hz}$ \\
\hline Number of samples & 2048 \\
\hline Frequency resolution & $1 \mathrm{~Hz}$ \\
\hline Nyquist frequency & $1024 \mathrm{~Hz}$ \\
\hline
\end{tabular}

Table 2: Properties of the simulated data set

\begin{tabular}{|l|l|l|l|l|l|}
\hline Mod & Resid & Natural & Acceptab & Dampi & Acceptab \\
\hline
\end{tabular}

\begin{tabular}{|l|l|l|l|l|l|}
\hline e & ue & $\begin{array}{l}\text { Frequen } \\
\text { cy }\end{array}$ & $\begin{array}{l}\text { le } \\
\text { Percenta } \\
\text { ge Error } \\
(\%)\end{array}$ & $\begin{array}{l}\text { ng } \\
\text { Ratio } \\
(\%)\end{array}$ & $\begin{array}{l}\text { le Percenta } \\
\text { ge Error } \\
(\%)\end{array}$ \\
\hline 1 & 10 & 128.0 & 2.344 & 0.300 & 16.667 \\
\hline 2 & 20 & 256.0 & 1.172 & 0.250 & 20.000 \\
\hline 3 & 30 & 512.0 & 0.586 & 0.139 & 35.971 \\
\hline 4 & 39 & 768.0 & 0.391 & 0.093 & 53.763 \\
\hline 5 & 50.7 & 806.4 & 0.372 & 0.046 & 108.696 \\
\hline
\end{tabular}

Table 3: List of SNR

\begin{tabular}{|l|l|}
\hline Situation & SNR \\
\hline 1 & 0.1 \\
\hline 2 & 1 \\
\hline 3 & 10 \\
\hline 4 & 30 \\
\hline 5 & 60 \\
\hline 6 & 100 \\
\hline
\end{tabular}

From previous work, the acceptable value used for $\mathrm{MCF}$ was greater than 0.9 and the acceptable number of identifications model, $N$ is around 70 to 80 . Thus $N=80$ is used throughout this study. It is also important to note that the acceptable percentage error for natural frequency and damping ratio is different. Moreover the percentage may vary for each mode for both parameters. For the purpose of this research, more restricted limits than previous work were defined where for natural frequency, deviation as much as $\pm 3 \mathrm{~Hz}$ from the true value is used as the limit. For damping ratio, maximum data deviation of \pm 0.05 will be applied. Any value greater than these is considered erroneous. Table 2 shows a list of acceptable percentage error at each mode for both parameters.

Further analysis will look upon the impact of following aspects towards the results:

1) The effect of shifting time interval

2) The effect of noise

3) Relationship between shifting time interval and quantity of selected data together with its impacts

4) The effect of data distributions towards the results 


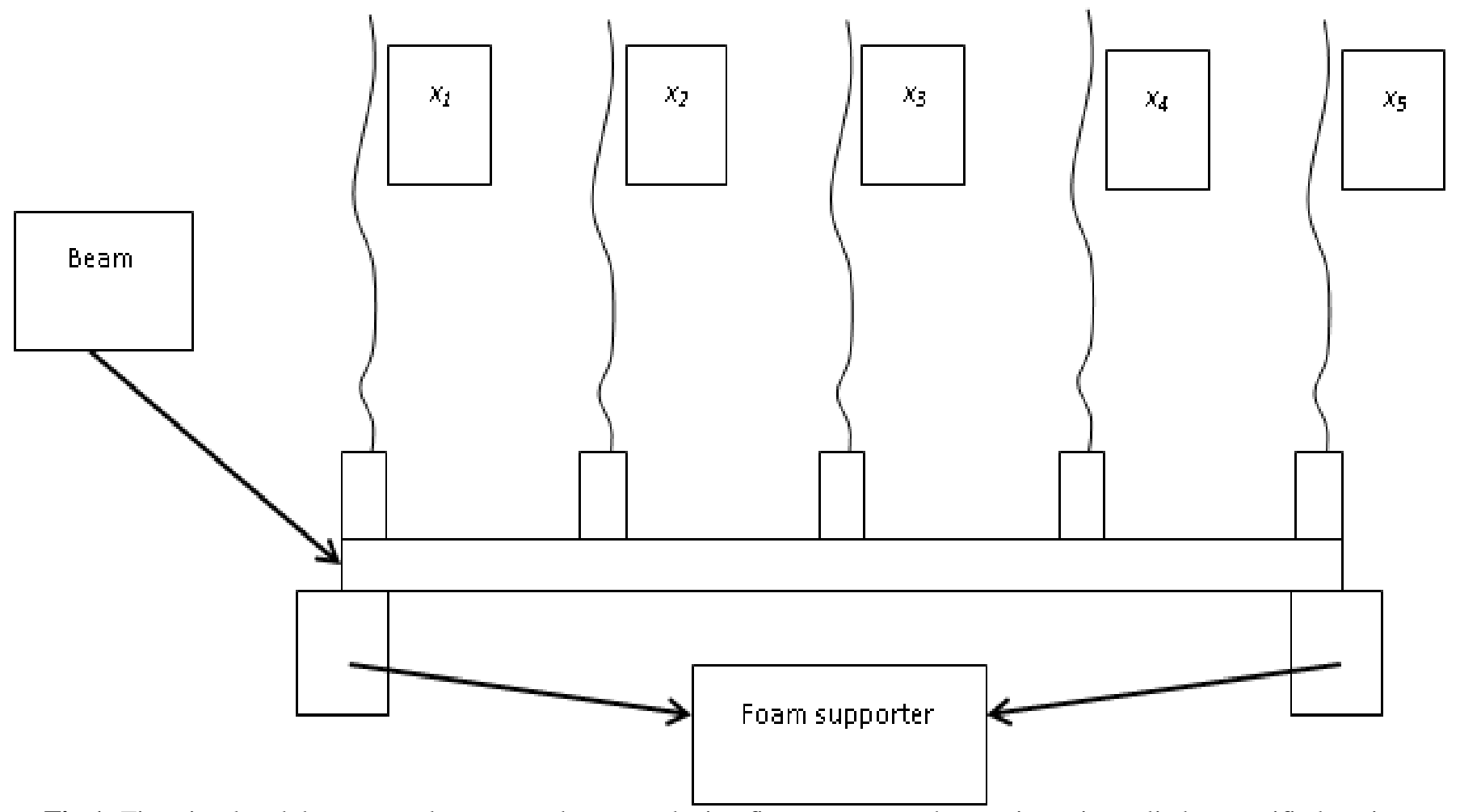

Fig 1: Five simulated data are analogous as a beam producing five responses when an input is applied at specific location.

\section{RESULTS AND DISCUSSIONS}

\subsection{Results for Natural Frequency}

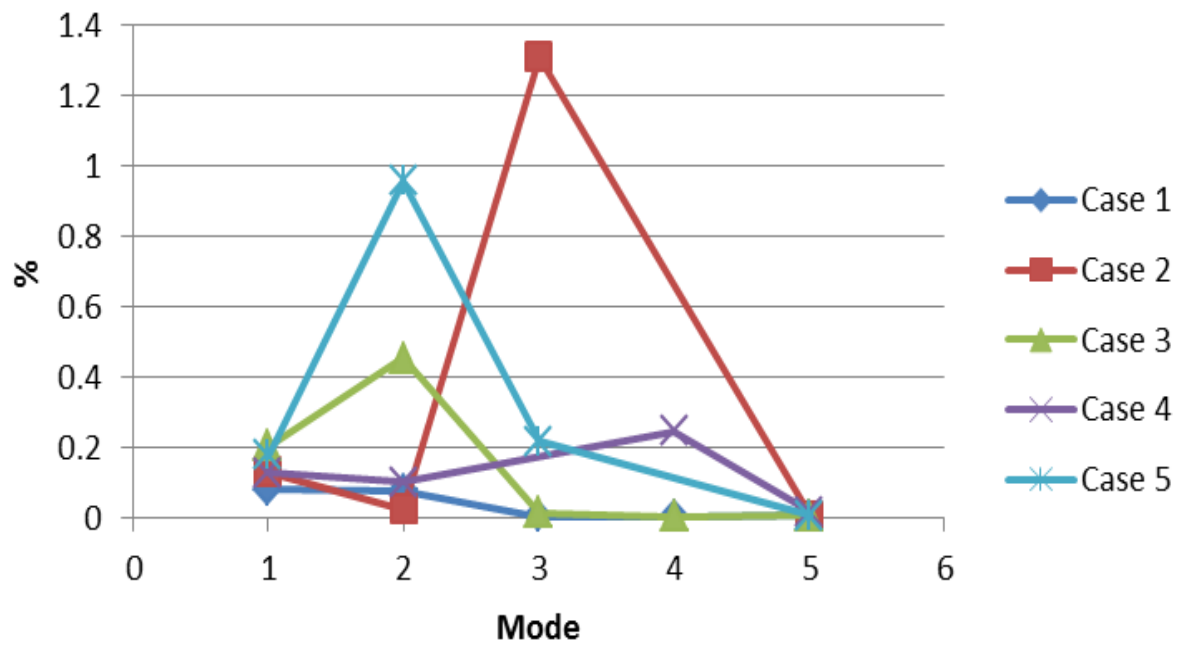

(a) 

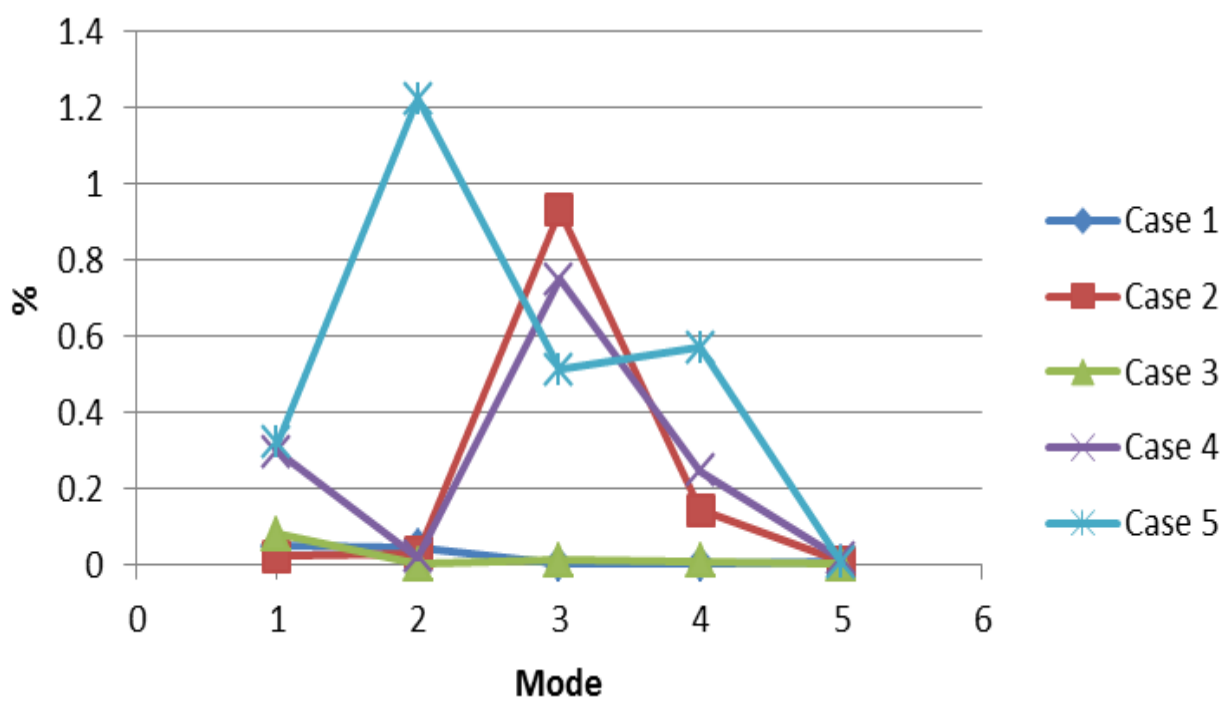

(b)
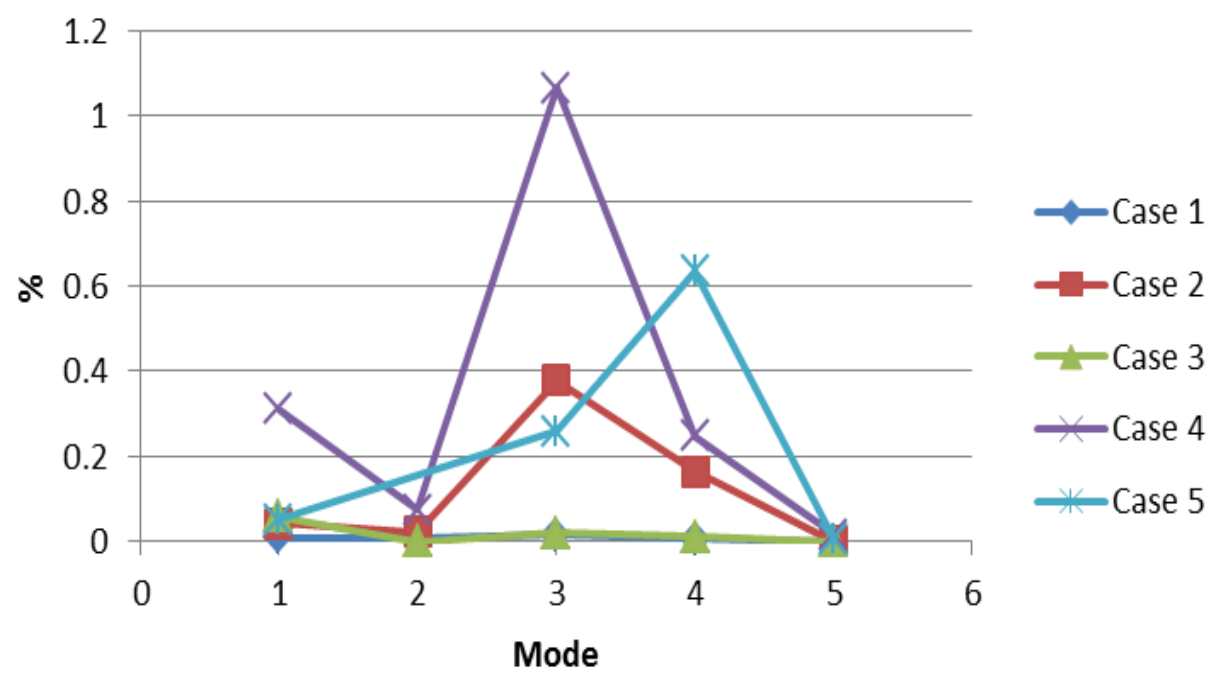

(c)
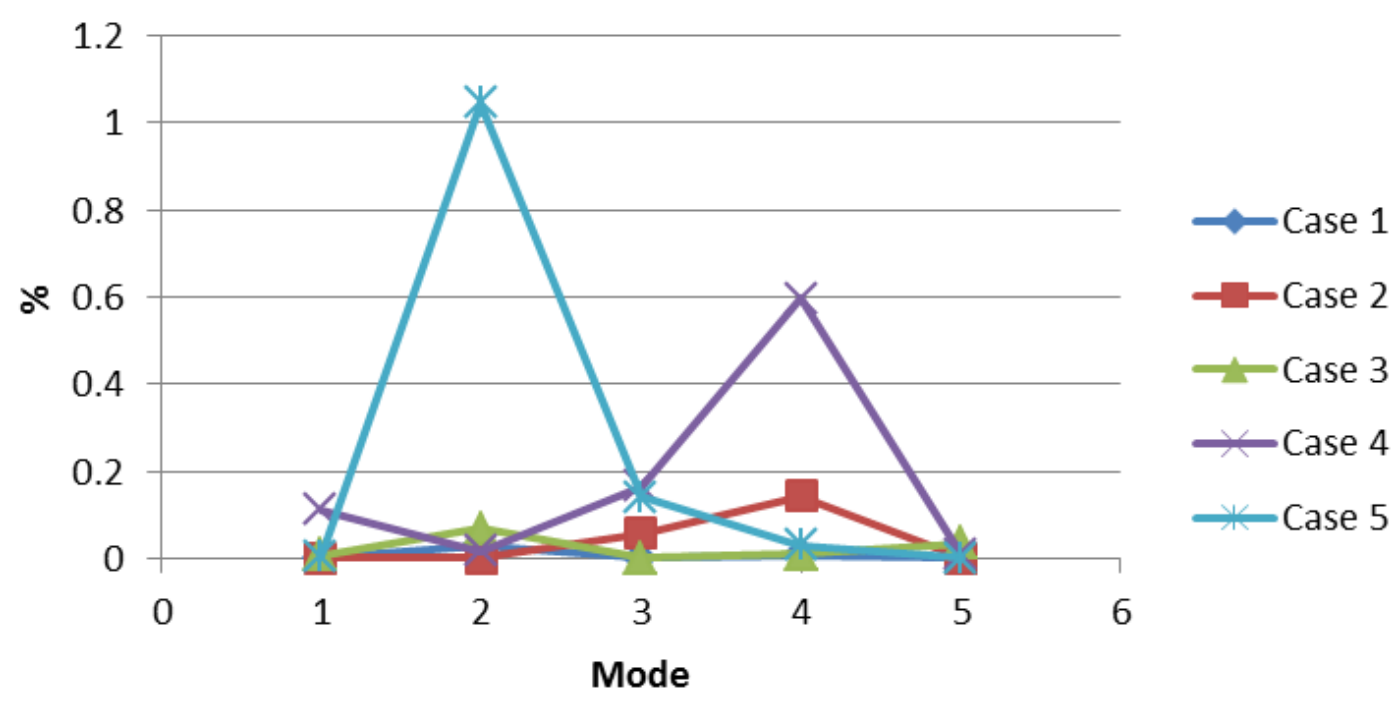

(d) 

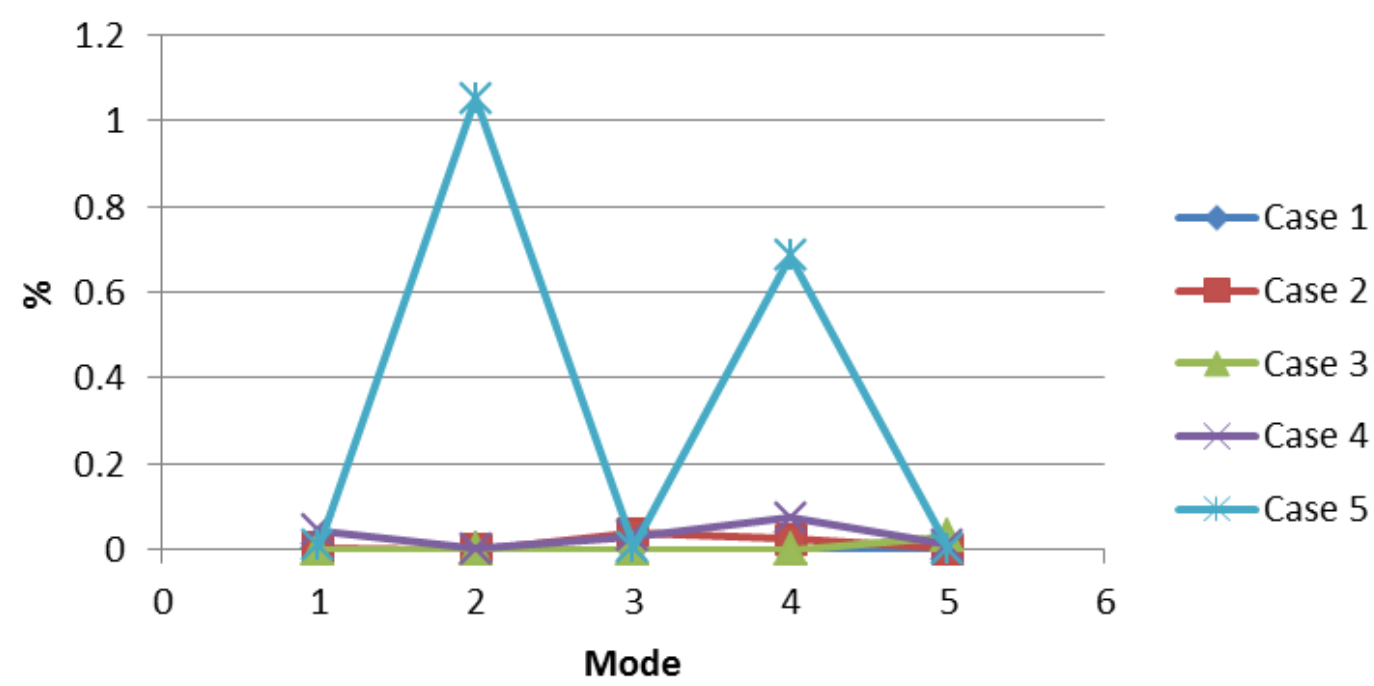

(e)
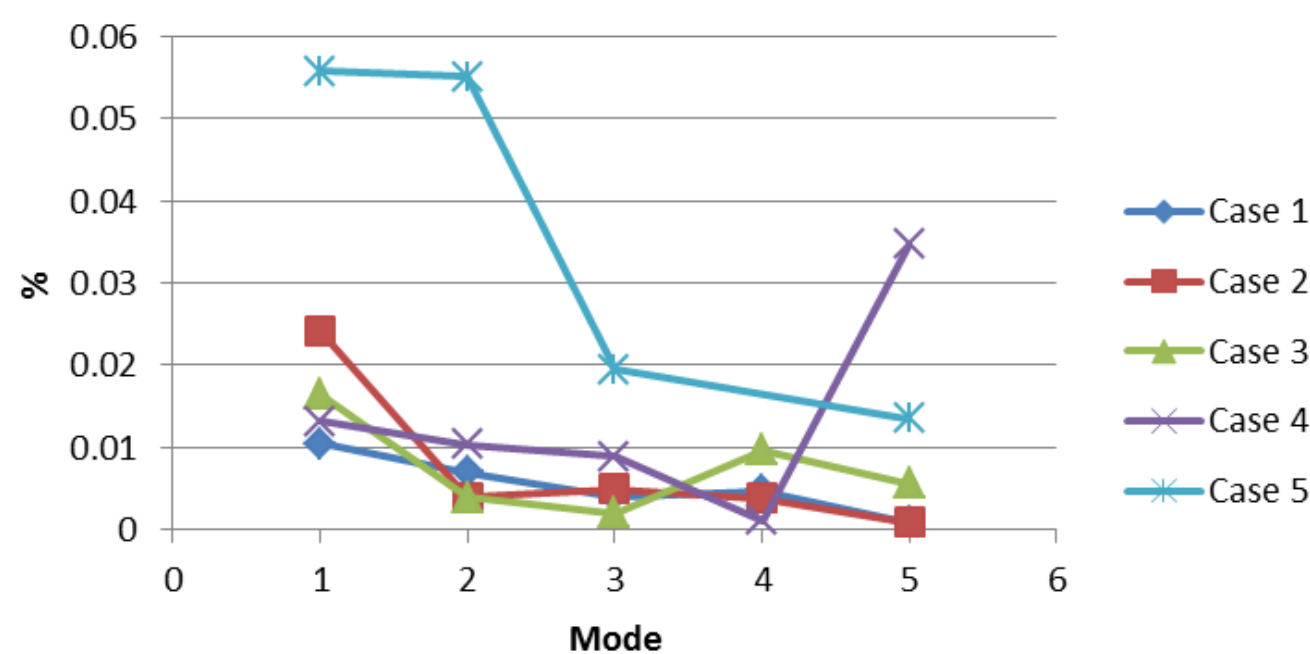

(f)

Fig 2: Natural frequency percentage error for all cases (a) SNR 0.1 (b) SNR 1 (c) SNR 10 (d) SNR 30 (e) SNR 60 (f) SNR 100

The discussions start by looking at the effect of shifting the time interval that occurs at the strongest signal, SNR 100. This is to ensure that the observation on the effect of time interval shift is not affected by the presence of noise. The comparison will made relative to Case 1 as it is the default settings in SSTD algorithm.

From Fig. 2-f, it can be seen that the results for all cases reside within the limit of acceptability as outlined in Table 2. Case 5 have the highest percentage error among all cases and there is one mode undetected by this case which is Mode 4. For the remaining cases, the results were much closed to each other. If taking the results for Case 1 as a point of reference for comparison, the results for Case 2, 3 and 4 fluctuated around the results for Case 1. For example, the results for Case 2 is higher than Case 1 for Mode 1, lower at Mode 2, slightly higher at Mode 3 before becoming lower back at Mode 4 and apparently same for Mode 5. The same pattern is also experienced by Case 3 and Case 4 .
The results also stressed out that Case 1 has the most consistent results in keeping low percentage for each mode for this situation. This is illustrated with a downward trend in the graphs from one mode to another. Cases such as Case 3 and 4 has upward trend after experiencing downward direction. If considering all cases, a small conclusion can be drawn where in general, the larger the gap in time interval, the higher the percentage errors will be. The ultimate errors were depicted by the results of Case 5. These findings confirmed and validated the pattern of graph for each case in the previous research work where it is similar to this study.

The following discussions will take account the presence of noise in SNR 0.1 until SNR 100. In a nutshell, the majority of the results for all cases meet the limits defined. From Fig. 2 , it can be seen that some of the cases did not detect some of the modes in the data. This is obviously shown in situation SNR 0.1 where Case 2, Case 4 and Case 5 did not detect mode 4 , mode 3 and mode 4 respectively. However the issue is reduced when the value of SNR is increasing 
except for Case 5 as stated earlier. This phenomenon highlights an attention where the amount of noise contained in the data do gave impact towards the results especially for cases that have shifted time interval.

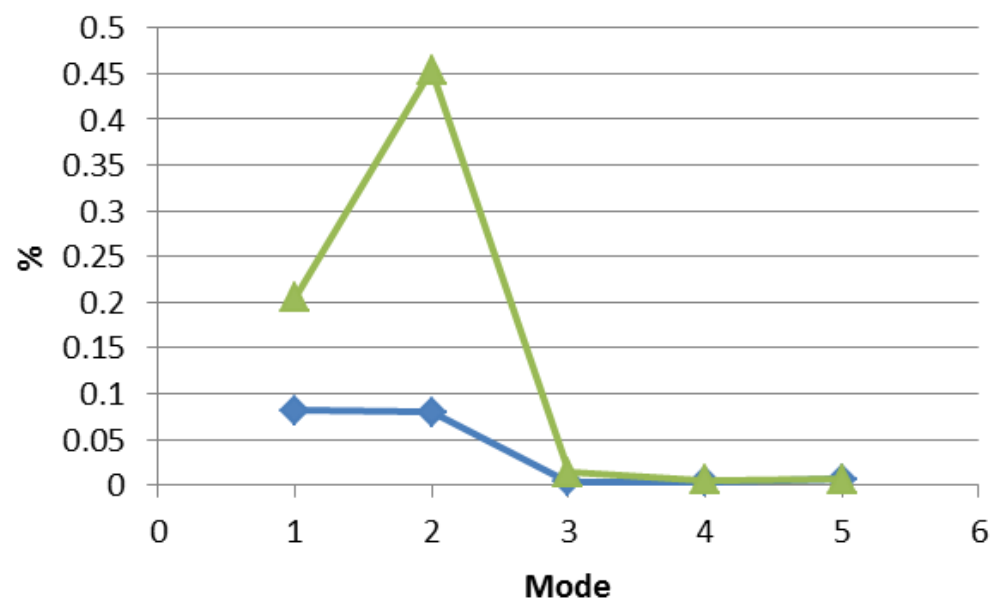

(a)

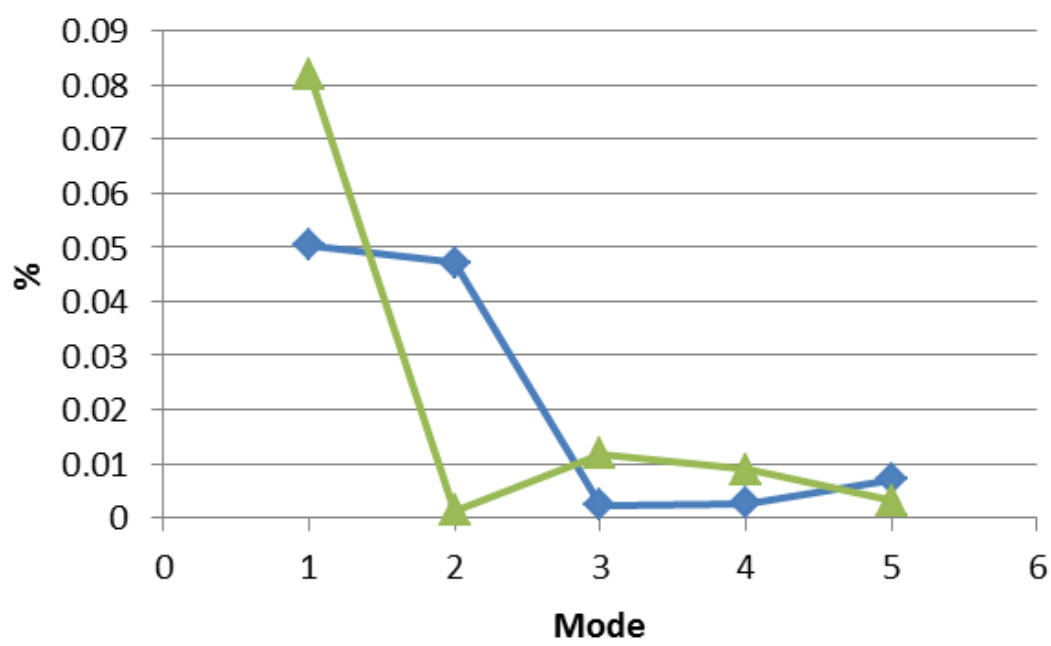

(b)

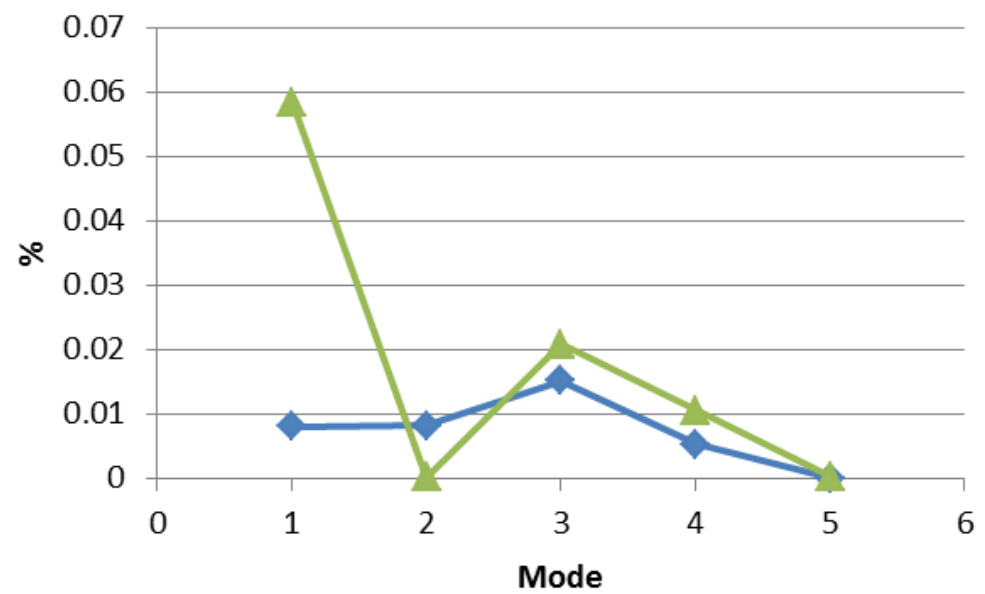

(c)
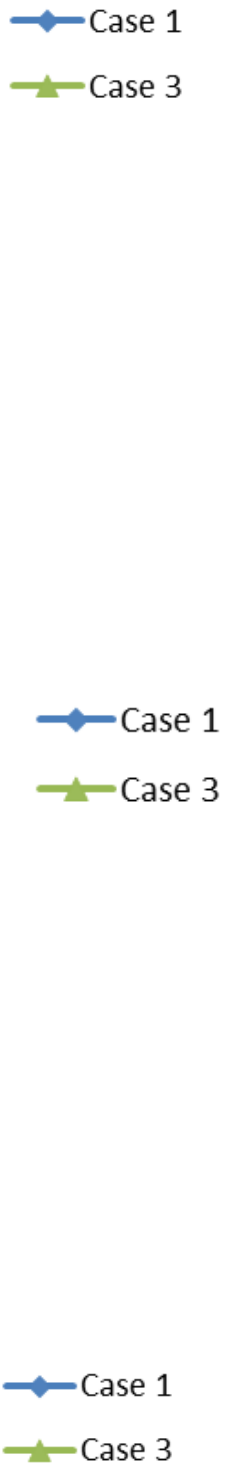


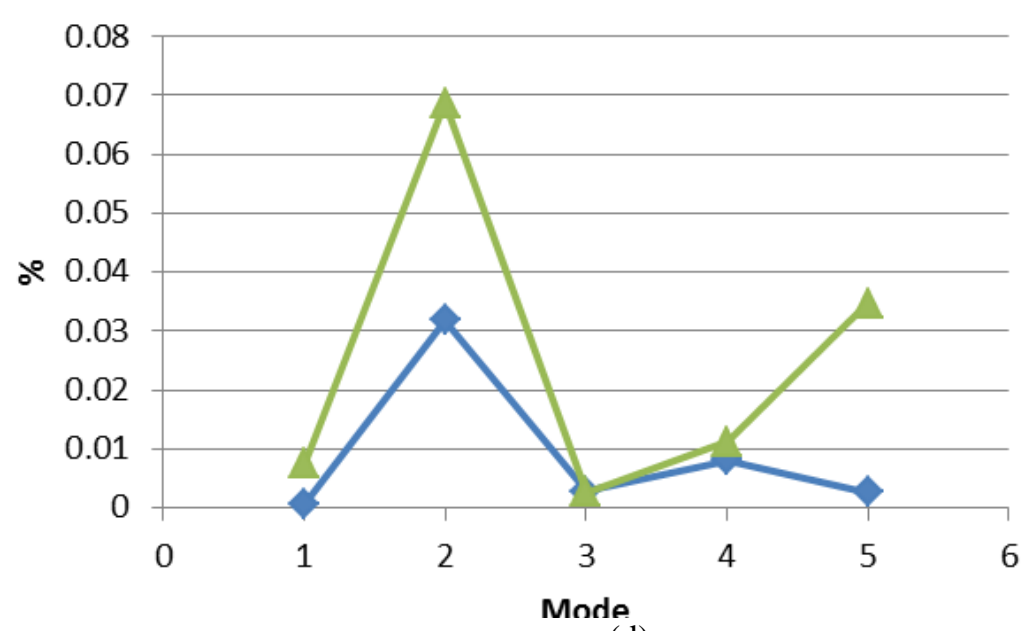

(d)

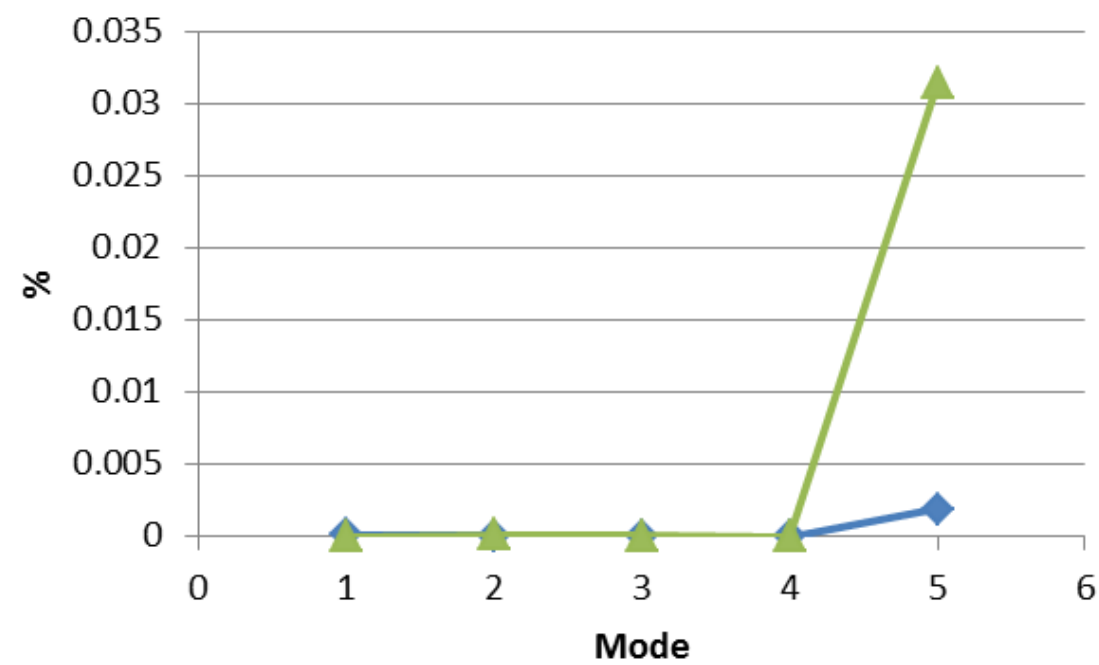

(e)

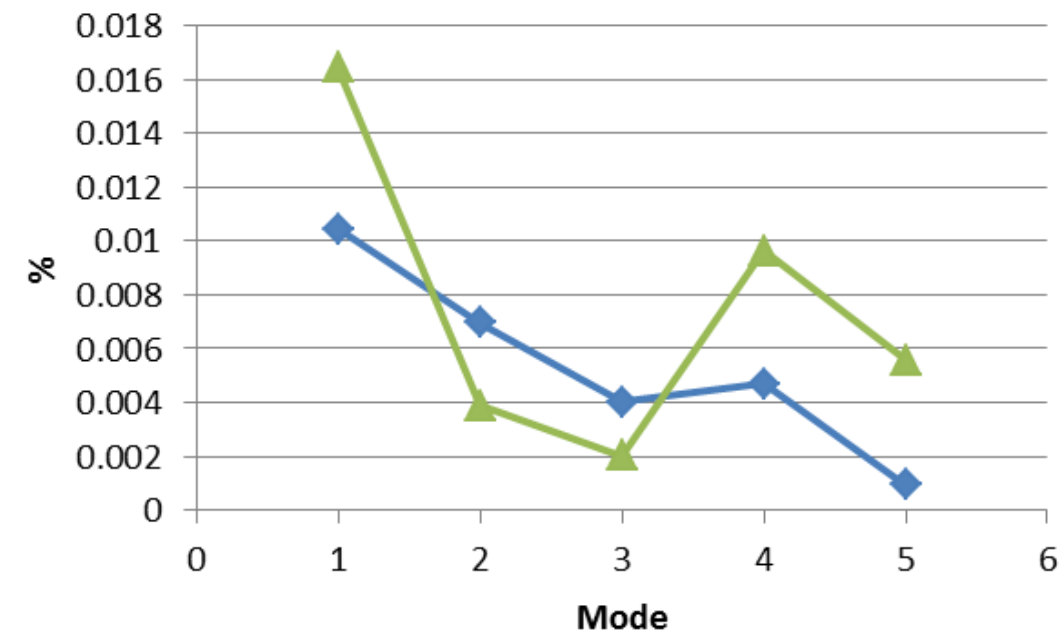

(f)

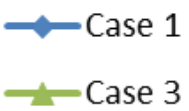

$\multimap$ Case 1

- Case 3

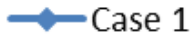

- Case 3

Fig 3: Natural frequency percentage error for Case 1 and Case 3 (a) SNR 0.1 (b) SNR 1 (c) SNR 10 (d) SNR 30 (e) SNR 60 (f) SNR 100

However, there is an exception for Case 3 as cases that always stay within the limits for all situations even when the signal is highly polluted with noise at SNR 0.1 are Case 1 and 3. If comparing these two cases only, Fig. 3 exhibits that Case 1 has lower percentage error than Case 3 for most of the times. This is in line with earlier statement mentioning 
about higher percentage error if the gap for time interval is increased. Hence, Case 1 and Case 3 are said to be robust with consistent low percentage errors for each mode for all situations including high presence of noise condition in comparison to other cases with the results for Case 1 better than Case 3 for almost all the time.

Also, it is essential to notify that most of the modes that clearly have a bit higher in percentage error relative to other modes as shown in Fig. 1 are Mode 3 and Mode 4 yielded by Case 2 and Case 4 . This is obviously seen in situation from SNR 0.1 to SNR 60 for respective cases. Although the figures were small and indicated that the difference is not significant, this marked an important point in next discussions. Finally, among all cases, Case 5 gave the most inaccurate result in terms of acceptability of percentage error and detection of mode.

\subsection{Results for Damping Ratio}

On the contrary, the percentage error results for damping ratio are higher than the results for natural frequency. These are expected as one of the reasons is due to mathematical situation where small deviation in numerator with respect to small value of denominator could sensitively lead to big difference (See Table 2). The interest lies more on this parameter as it has long been the subject of research since Rayleigh introduced the well-known 'viscous damping'

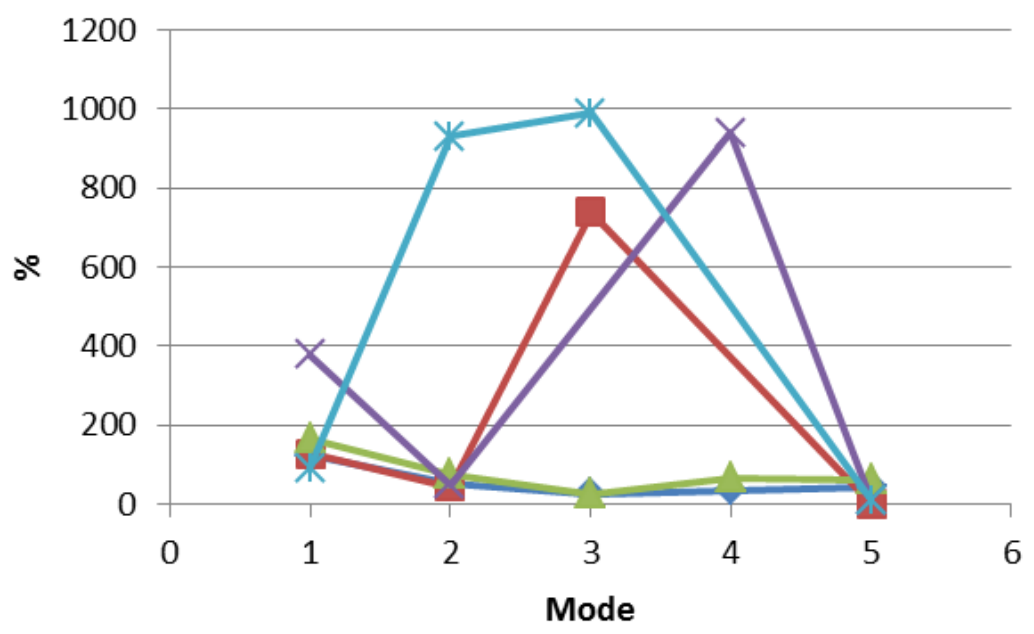

(a)

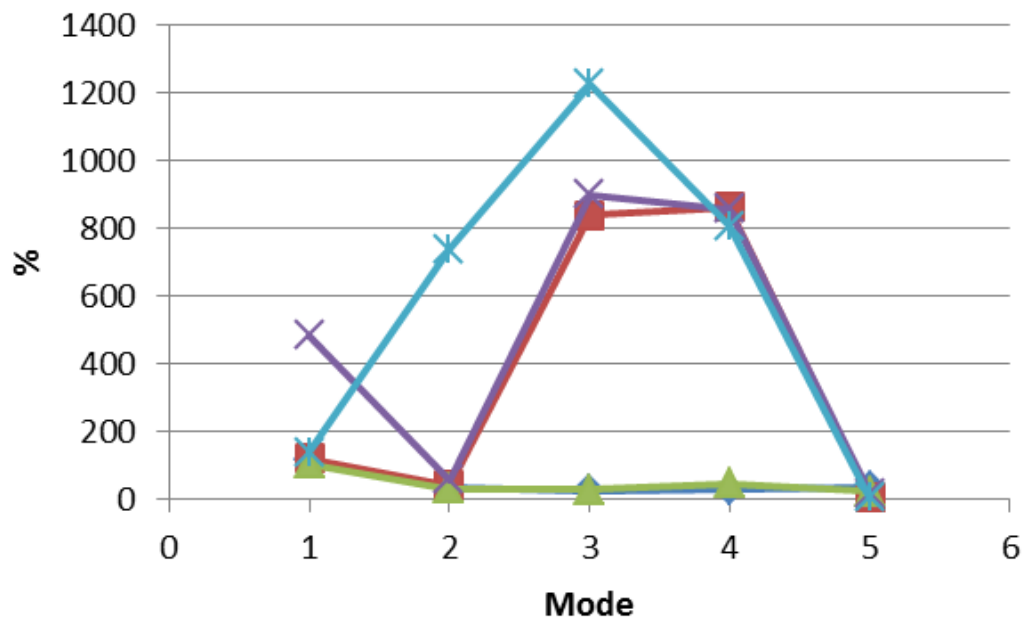

(b)
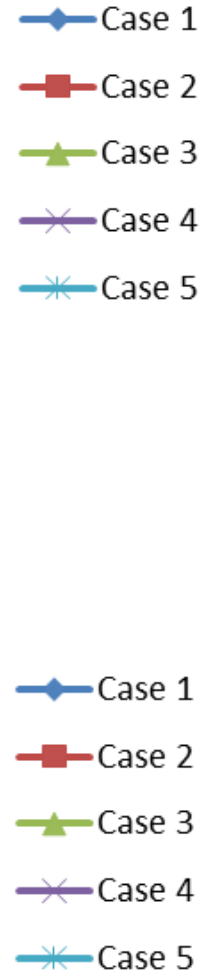

6 

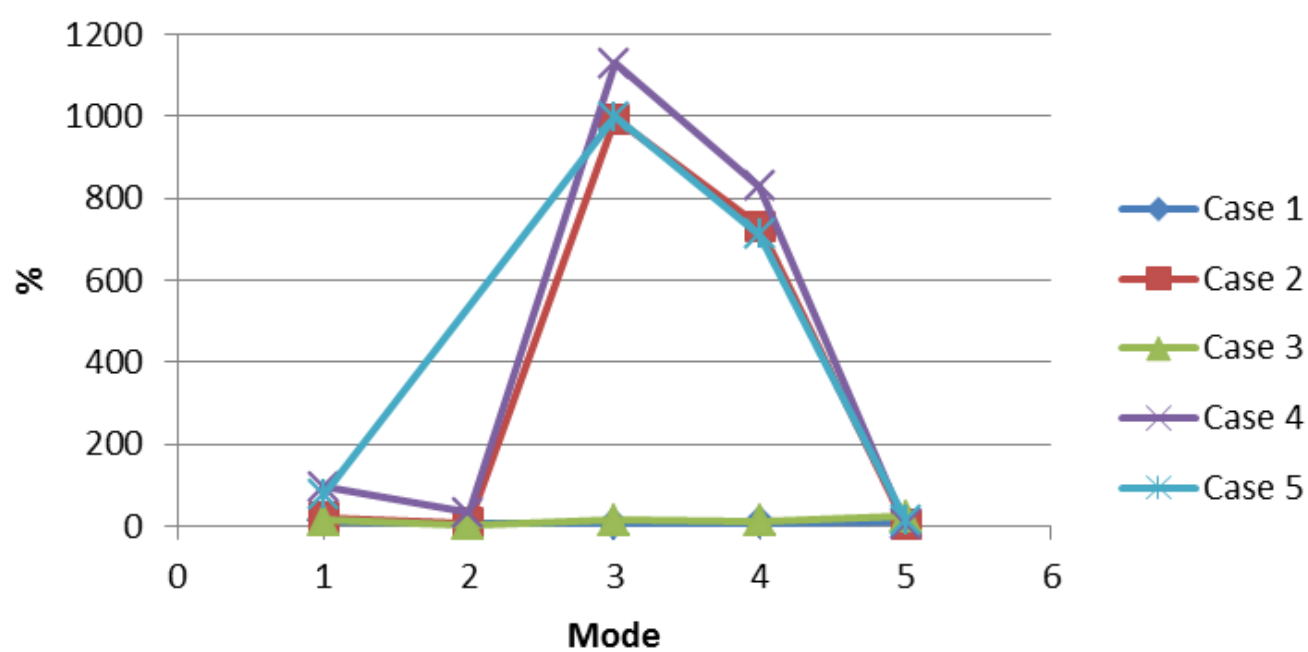

(c)
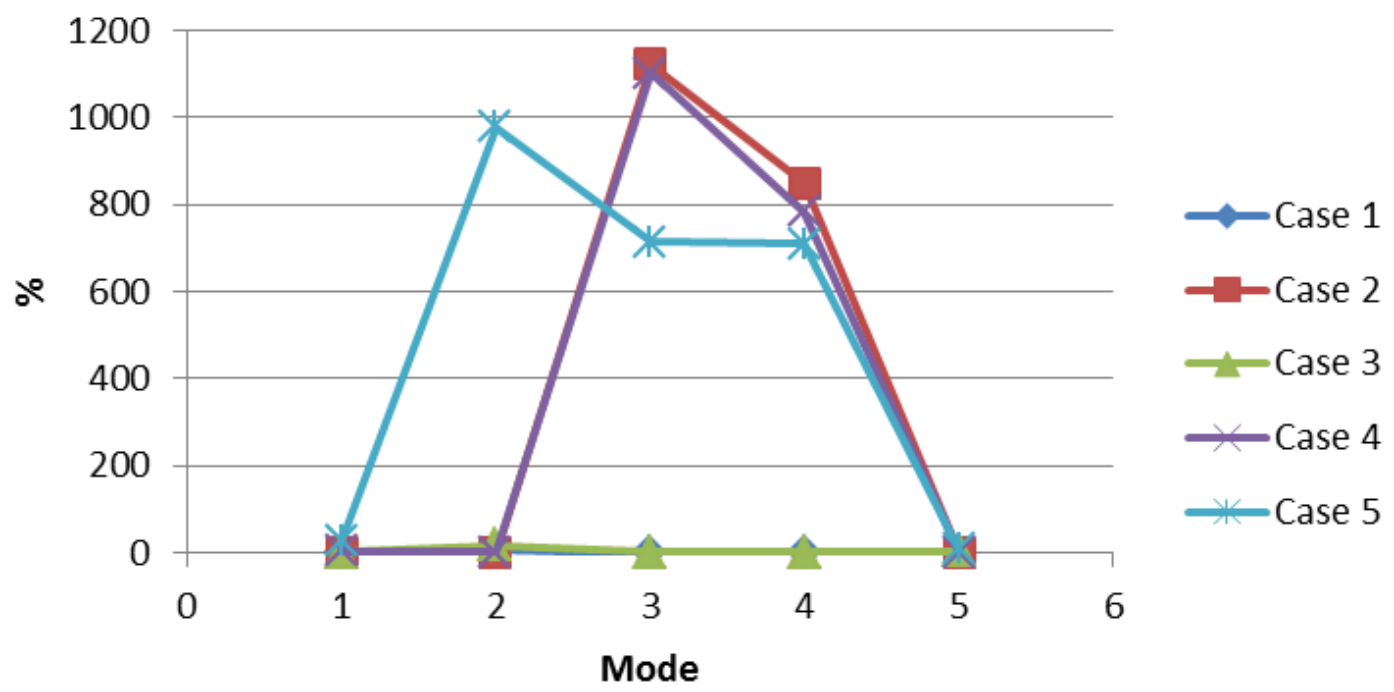

(d)

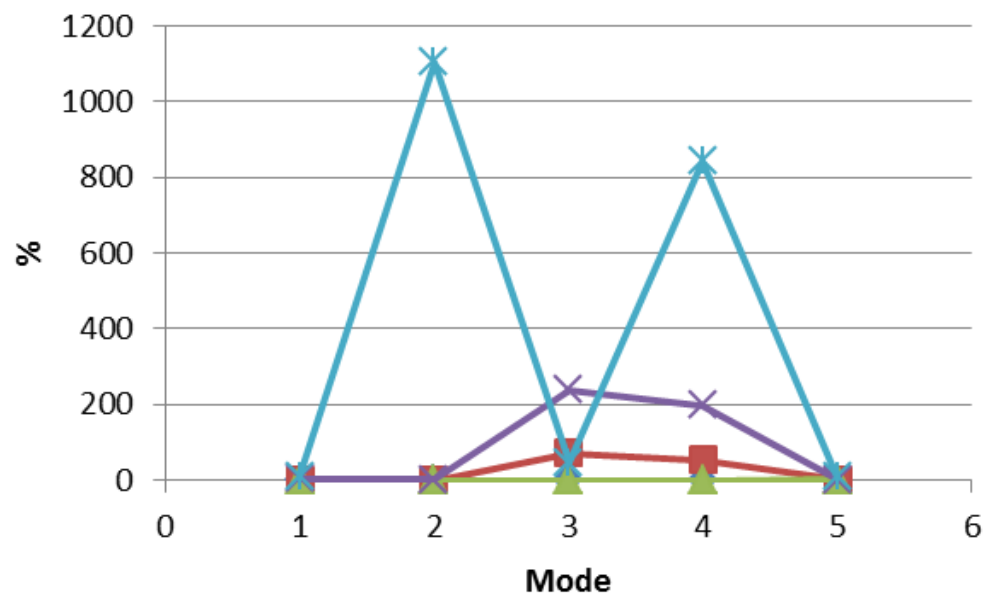

$\leadsto$ Case 1

- Case 2

$\rightarrow$ Case 3

$\longleftarrow$ Case 4

* Case 5

(e) 


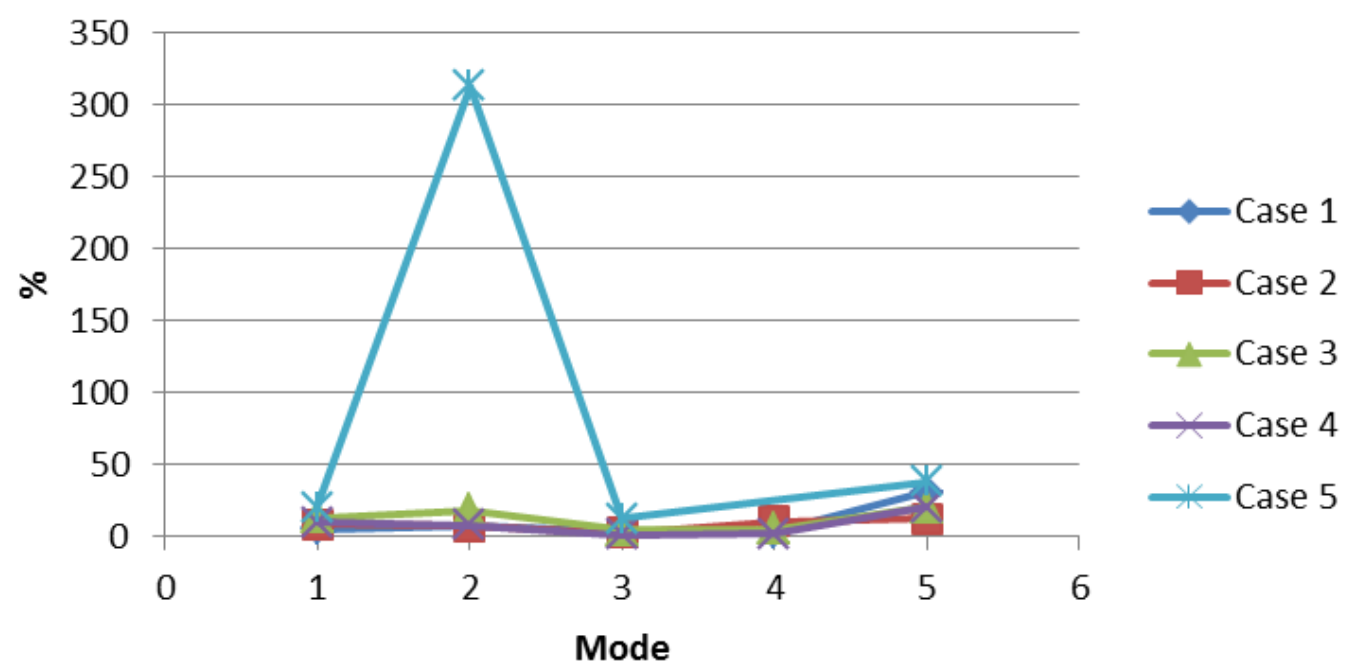

(f)

Fig 4 Damping ratio percentage error for all cases (a) SNR 0.1 (b) SNR 1 (c) SNR 10 (d) SNR 30 (e) SNR 60 (f) SNR 100

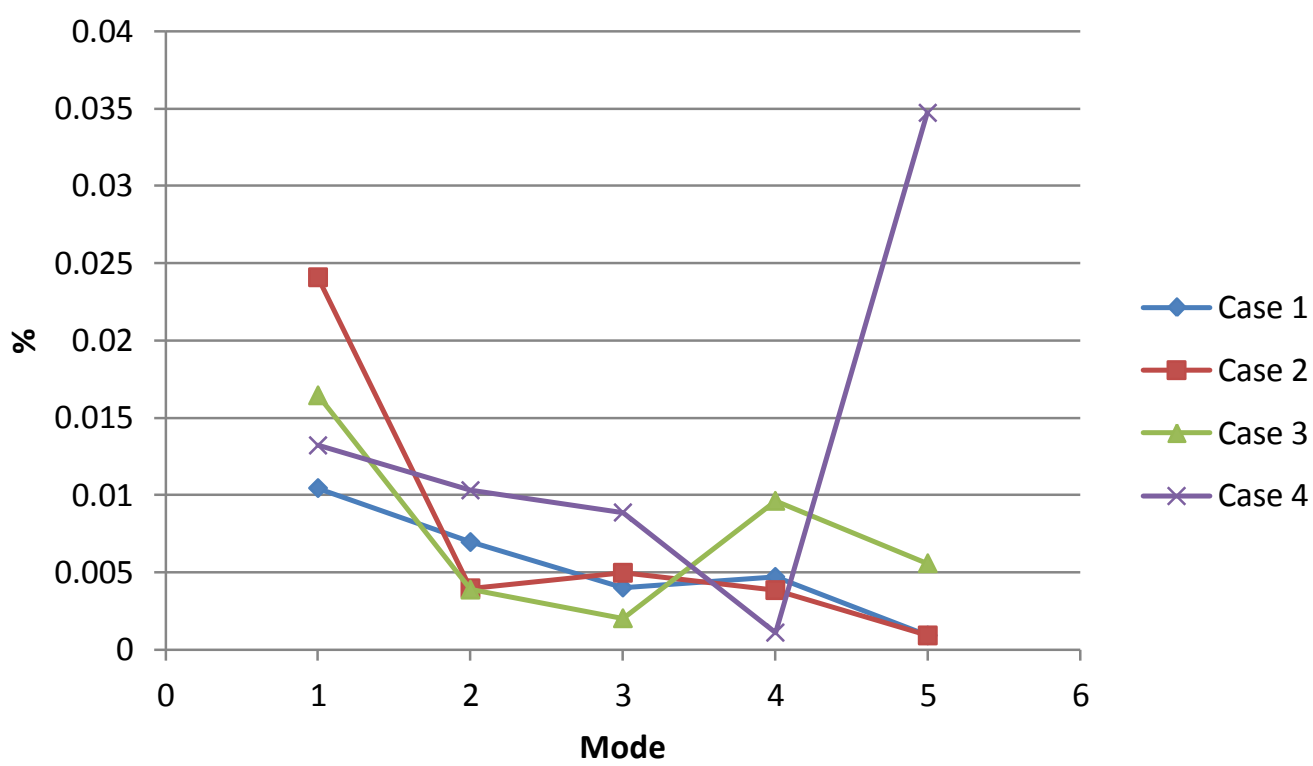

Fig 5 Damping ratio percentage error for Case 1, Case 2, Case 3 and Case 4 for SNR 100

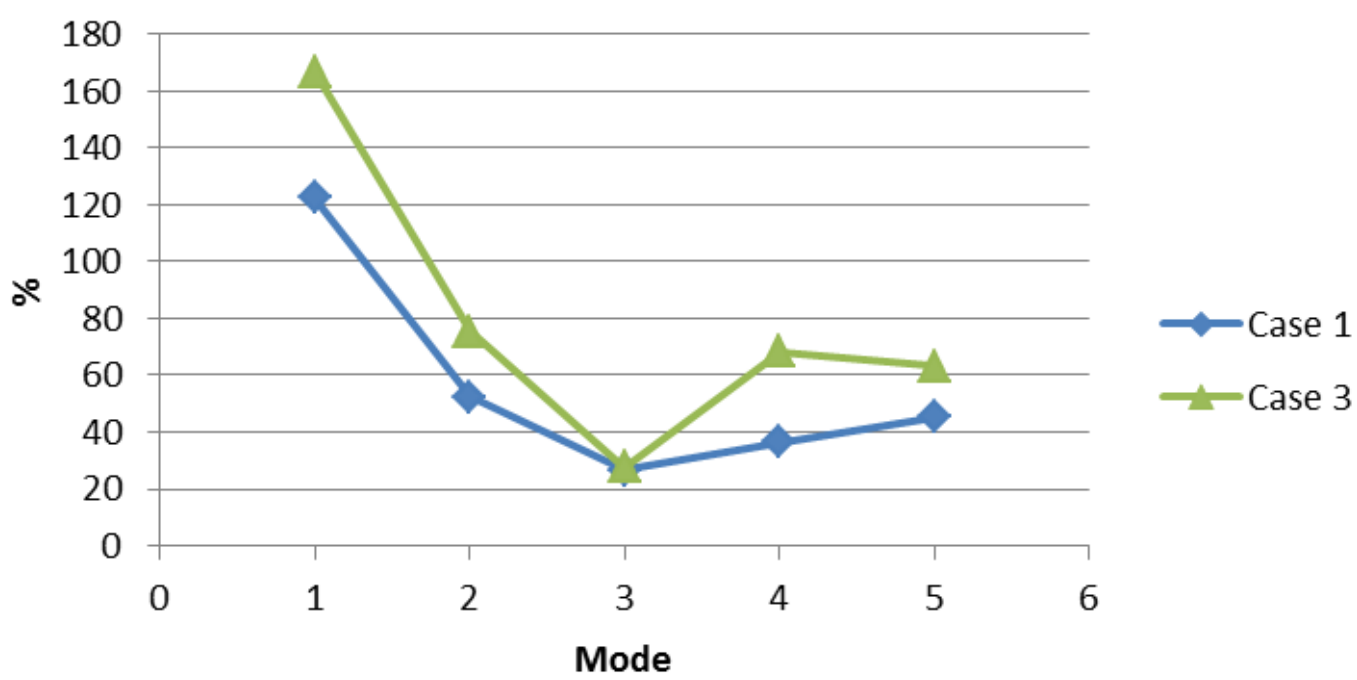


(a)
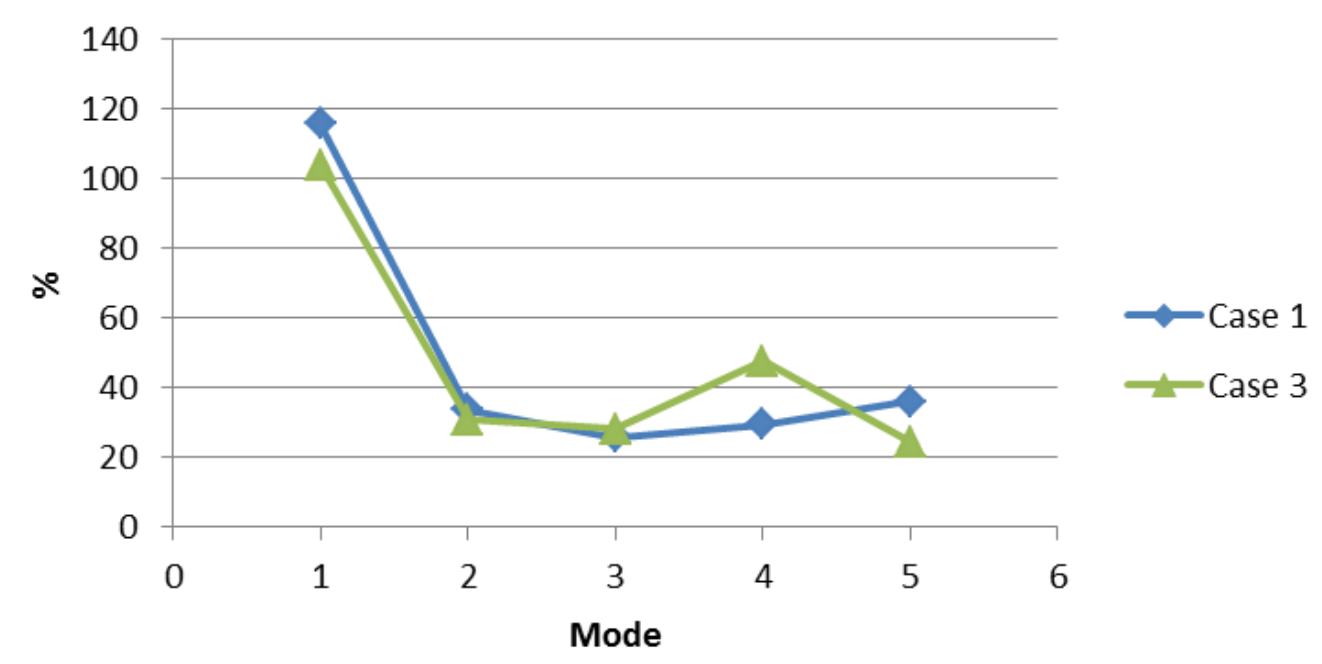

(b)

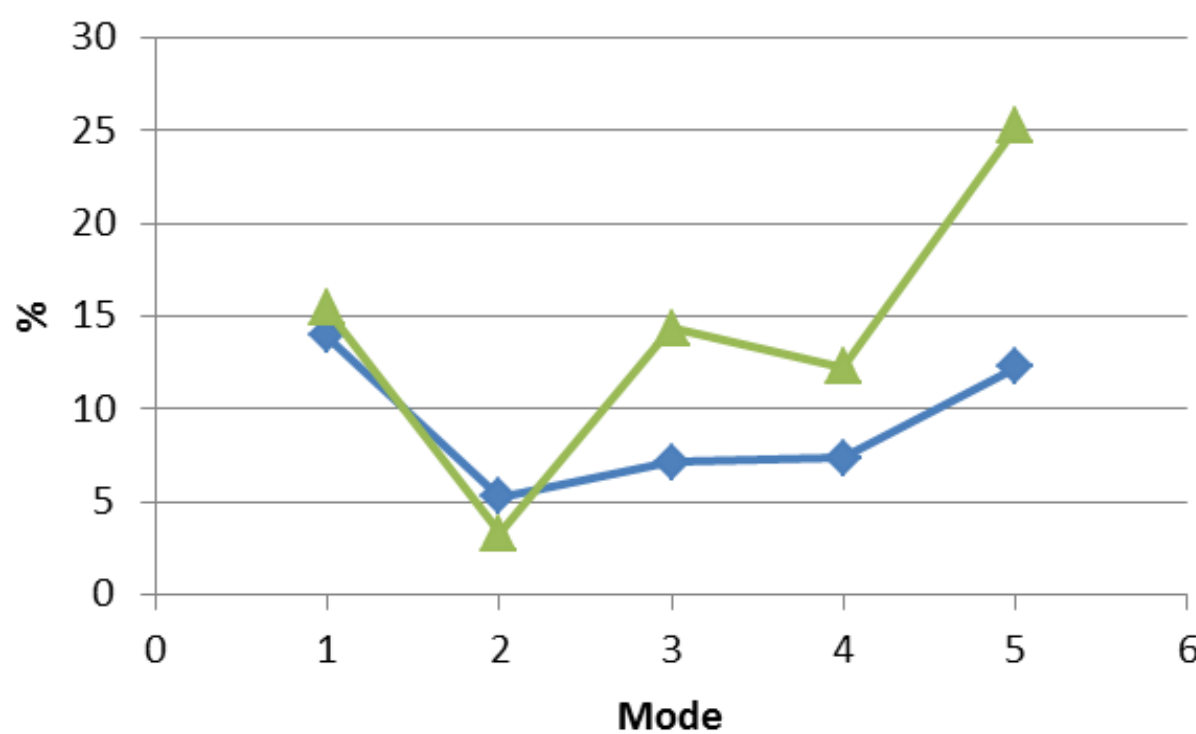

(c)

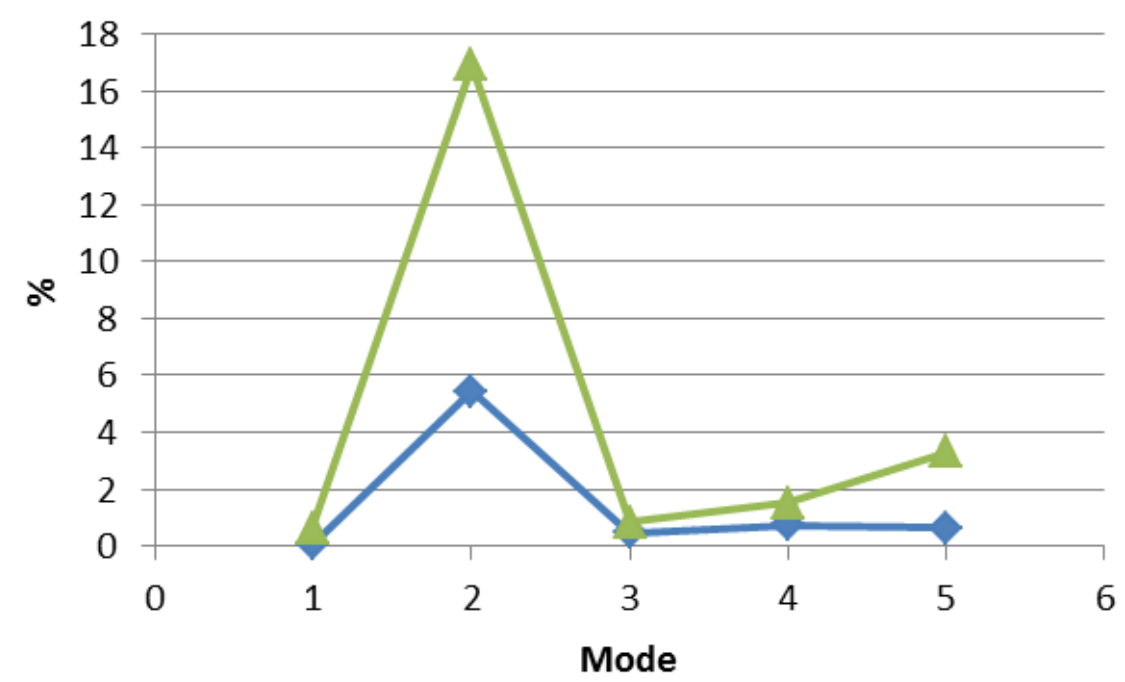

$\sim$ Case 1

- Case 3

(d) 

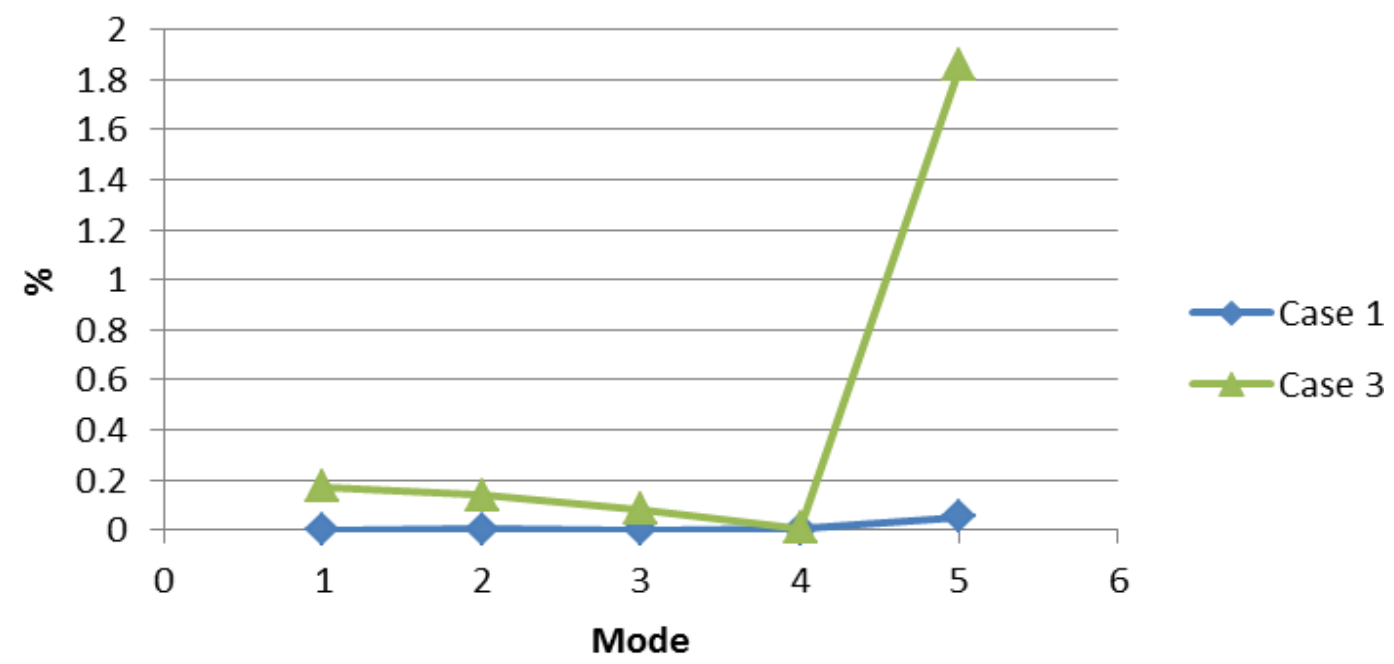

(e)
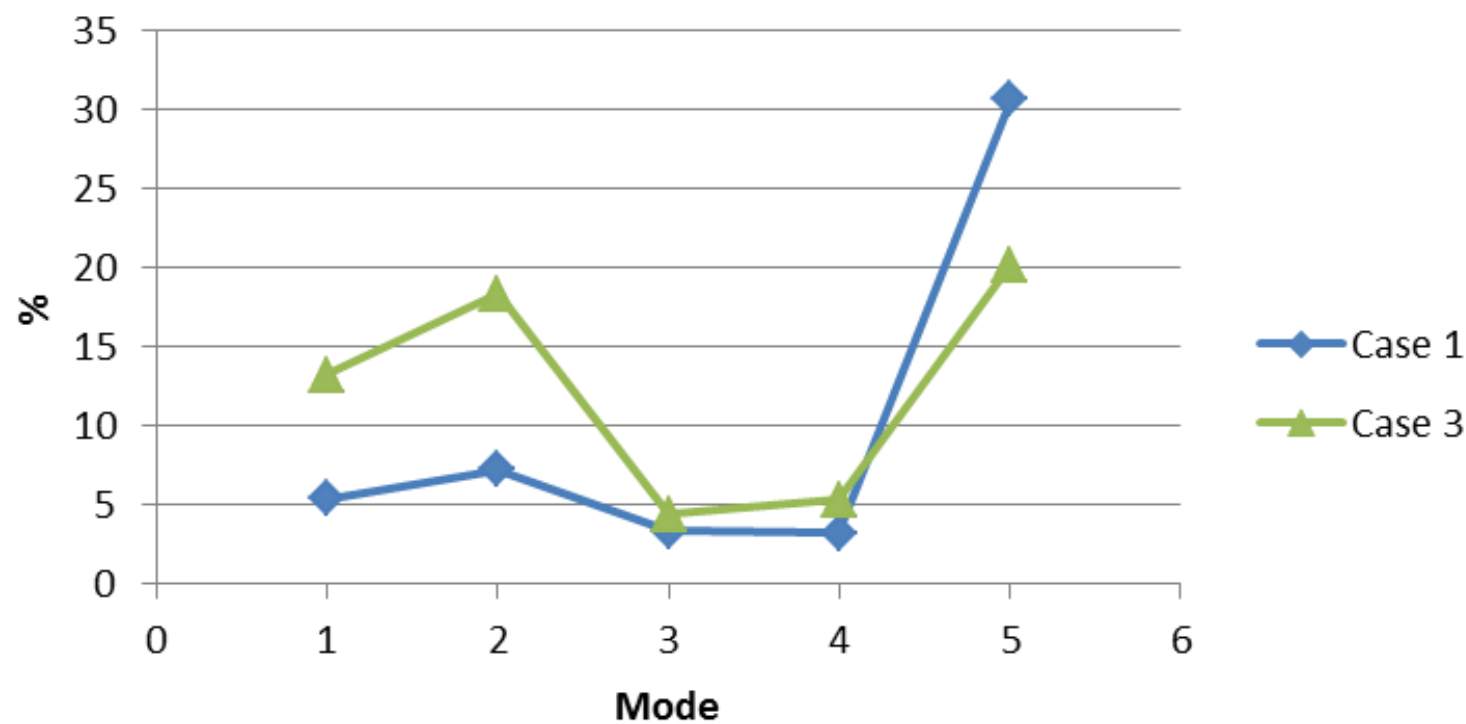

(f)

Fig 6 Damping ratio percentage error for Case 1 and Case 3 (a) SNR 0.1 (b) SNR 1 (c) SNR 10 (d) SNR 30 (e) SNR 60 (f) SNR

concept in 1877. However, the understanding of this parameter is still primitive until today in contrast to inertia and stiffness forces. This sparks an impetus to extensively conduct more research in fulfilling better understanding towards this parameter [23-26].

Similarly, the discussion begins by looking at the strongest signal, SNR 100. Generally, Fig. 4 portrayed that all cases have percentage errors within the limits introduced. An exception goes to Case 5 as it did not identify Mode 4 as it also did not detect for natural frequency parameter. Plus, there is also an obvious, over-exceeding result for Mode 2. These evidences indirectly make Case 5 to have the poorest result among all.

For the rest of the cases, findings showed that increasing the shift in time interval produced better results in comparison to Case 1 for certain mode. In previous work, Case 4 was proven to have the most consistent result in keeping low

\section{0}

percentage error for most of the modes. Whereas in this study, by assessing the results at each mode individually highlights Case 2 and Case 4 having promising potentials where they do have better results at specific mode in comparison to Case 1 . The results for Case 3 were also improved especially for higher modes. Hence, from this observation, the statement 'larger gap in time interval yield larger percentage error' only hold true for Case 5.

However, if the analysis considers the presence of noise starting from SNR 0.1 to SNR 100, some of these promising cases turned out to be very erroneous from the boundary agreed. Surprisingly in certain situations, the results were worse than Case 5 as illustrated in Fig. 4. Good examples were Case 2 and Case 4 themselves seen in each situation except for SNR 100. The over-exceeding errors are reduced as the amount of noise is also reduced. Some failure detection of mode in natural frequency also followed consecutively in damping ratio. Again, the phenomenon 
highlights the impact of noise towards damping ratio results especially for cases that have shifted time interval.

By inspecting closely at the results, it appears that only Case 1 and Case 3 reside within the limits defined for all situations except for SNR 0.1 and SNR 1. For these situations, the results only for Mode 1 and Mode 2 for both cases were drastically surpassed the limits. Fig 6 depicts that the erroneous results for Mode 1 were very high by merely triple in relative to the rest of the modes including Mode 2. It is suspected that this is primarily due to significant amount of noise in the signal. In general, the consistency on keeping low percentage for damping ratio achieved by Case 1 and Case 3 for each mode in almost all situations makes them robust among all cases. Again, comparing both cases only, Case 1 provided lower percentage error results than Case 3 for most of the times.

An important note to point out is the contrast results observed for the strongest signal situation, SNR 100 from the expected. It is stated earlier that the results improved as the amount of noise is reduced. This can be observed in both natural frequency as well as damping ratio parameter. However, for this situation, the percentage error is increased once again instead of decreasing relative to the results for SNR 60 in both cases. Moreover, the increment is higher than the results for SNR 10. Although this happen, the results were still within the limit. Nevertheless, if comparison is made with the previous work, approximately the same range of figures is obtained in both studies. A suggestion for repeating numerical experiment for both SNR 60 and SNR 100 is recommended.

Another observation that can be peeked is that for Case 2 and 4 , the over-exceeding result mostly occurred for Case 2 and Case 4 are Mode 3 and Mode 4. The errors were obvious where it can rise up to five times greater than percentage error for the rest of the mode. The same pattern is also observed in natural frequency results. The difference is that the figures were small and thus, the phenomena may not as obvious as in damping ratio results.

\subsection{Number of Data}

One way of looking the factors that give effects towards the results is to observe the number of data selected by the algorithm for each case. These data is then used to calculate the average result for both natural frequency and damping ratio parameters. Following discussions will imply the same previous format where it begins with SNR 100.

Fig. 6 depicts that Case 2 produced the largest number of data for almost every mode. This is then followed by Case 1, Case 3 or Case 4 and finally Case 5. It was mentioned earlier that Case 5 did not detect one mode for this situation which is Mode 4. The diagram spells out the reason where none of data were selected at that mode after MCF filter is applied in the algorithm. From these findings, a number of deductions can be made:

1) In general, the overall trend illustrated that as the shift in time interval is increased, the number of data selected will decrease. This evidence is showed by the ranking of cases according to the data quantity possessed by each case. The ultimate least number of data held by Case 5 agreed with this statement although the largest number of data was initially occupied by Case 2 instead of Case 1 .

2) The number of data is an indicator in assessing the accuracy of results but the quantity is not the sole indicator for this purpose. As a proof, the least percentage errors for damping ratio among all cases were held by Case 4 with 59 data. Whereas, Case 1 had the largest with 68 data but occupied second in terms of lowest percentage errors. This can be clearly seen in next example.

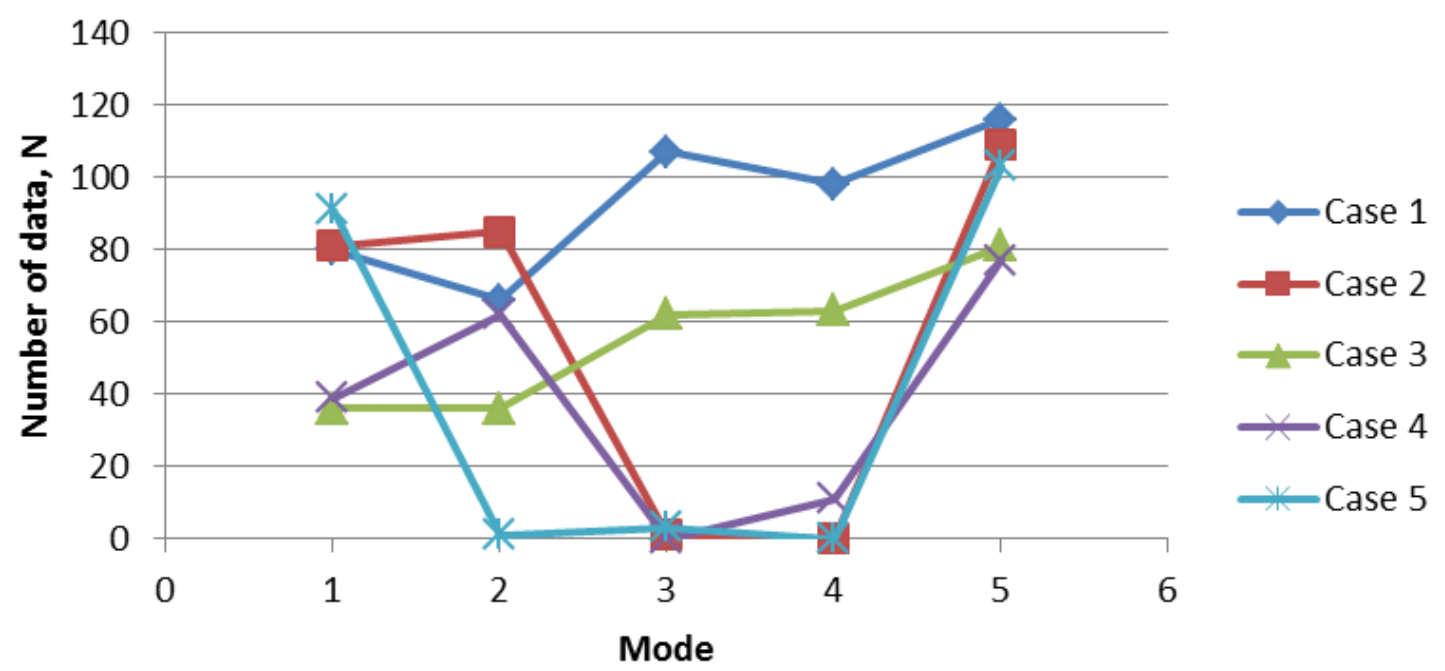

(a) 

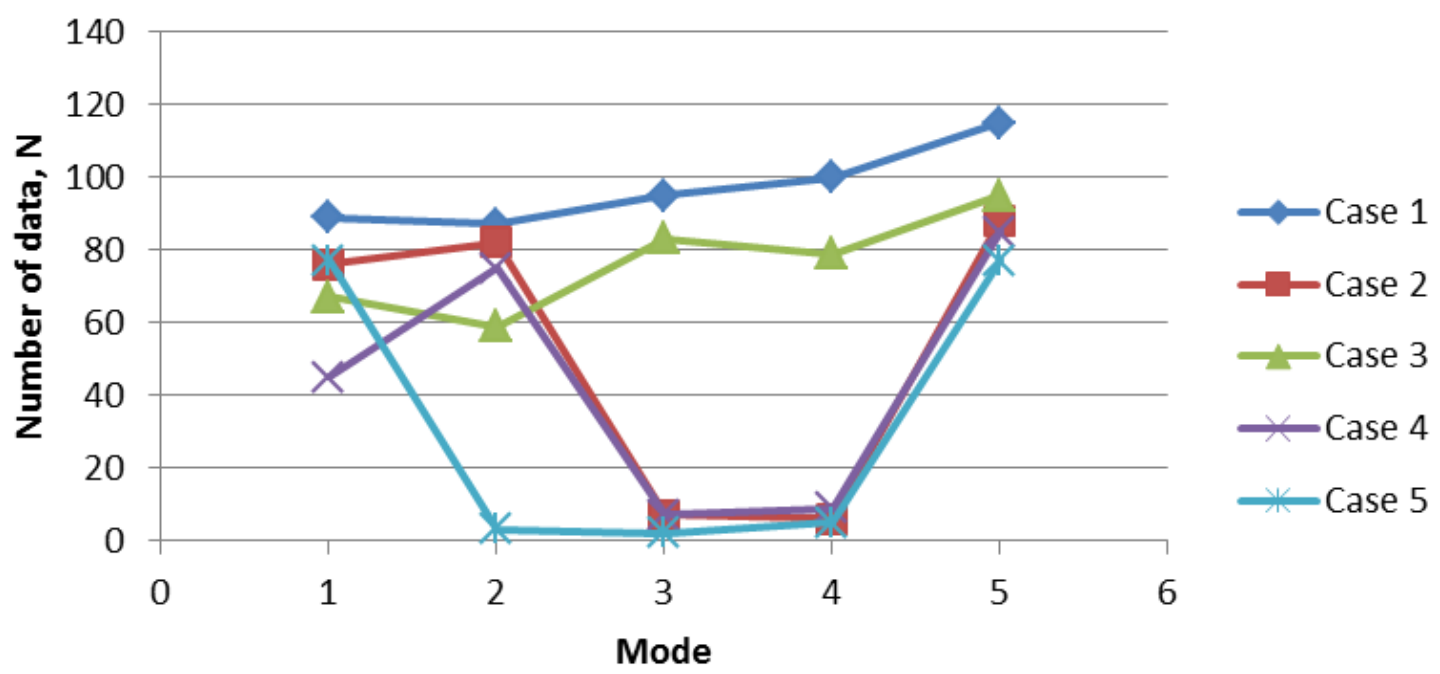

(b)
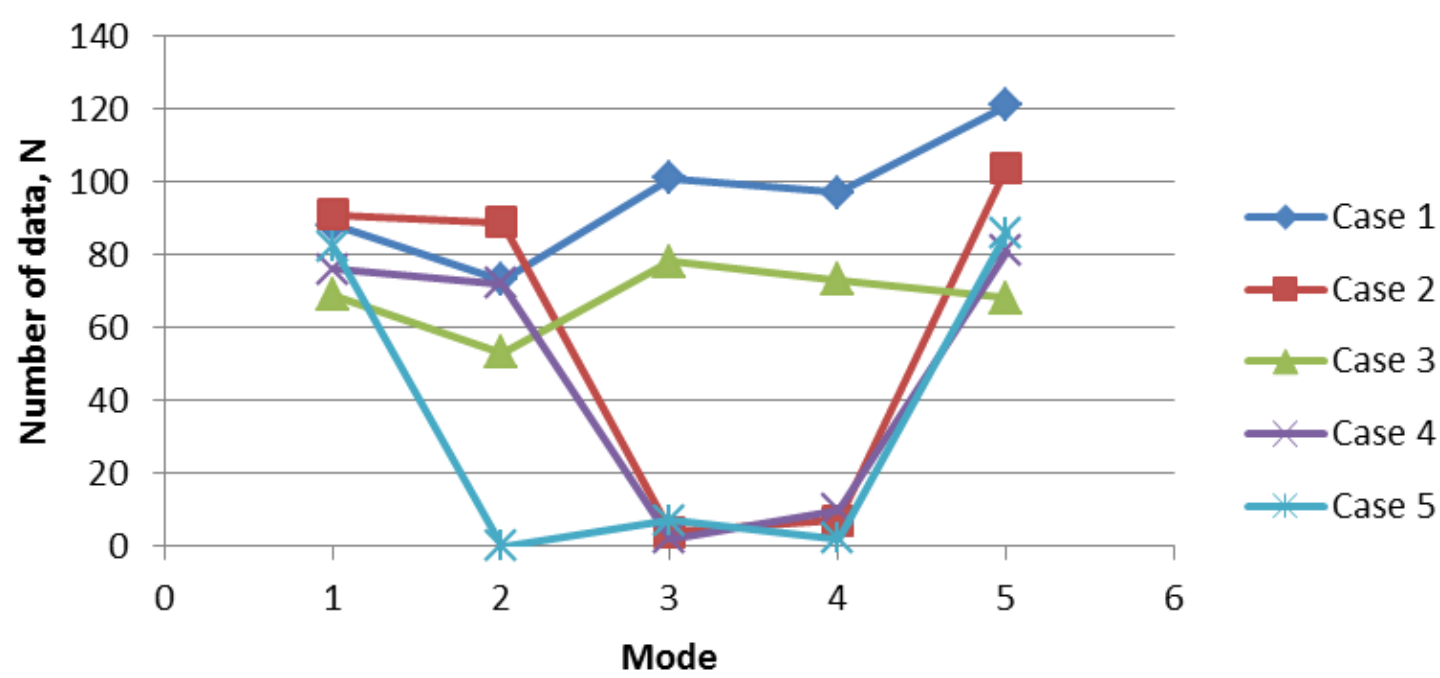

- Case 2

$\rightarrow-$ Case 3

$\leftarrow$ Case 4

* Case 5

(c)
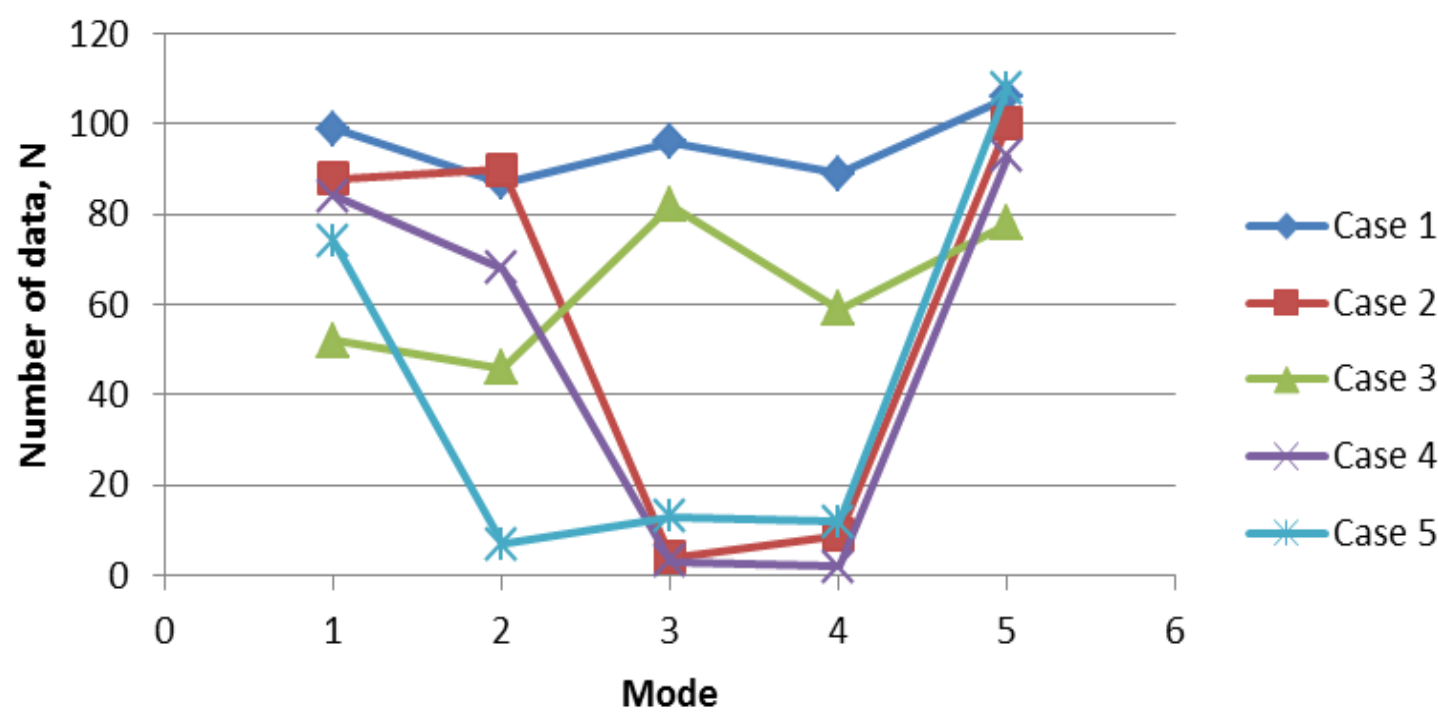

- Case 2

- Case 3

$\leftarrow$ Case 4

Case 5

(d) 

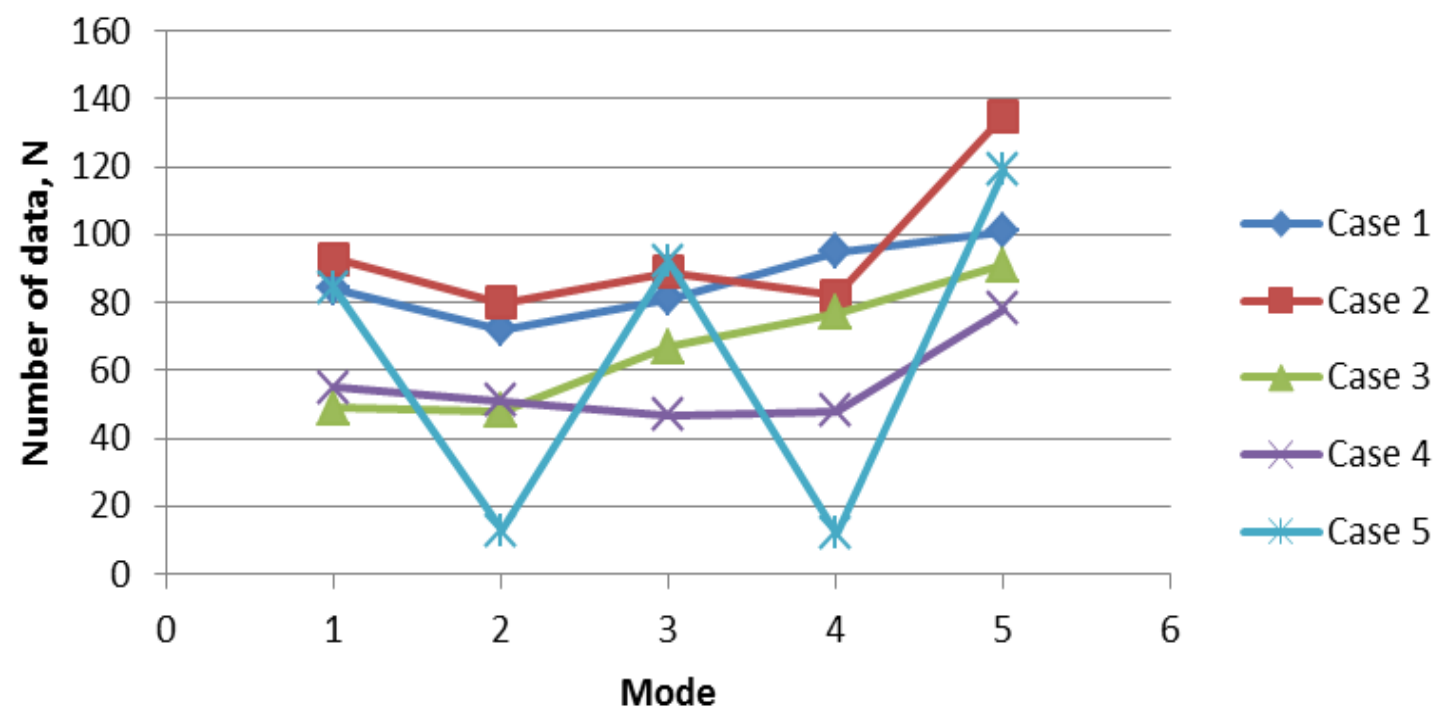

(e)
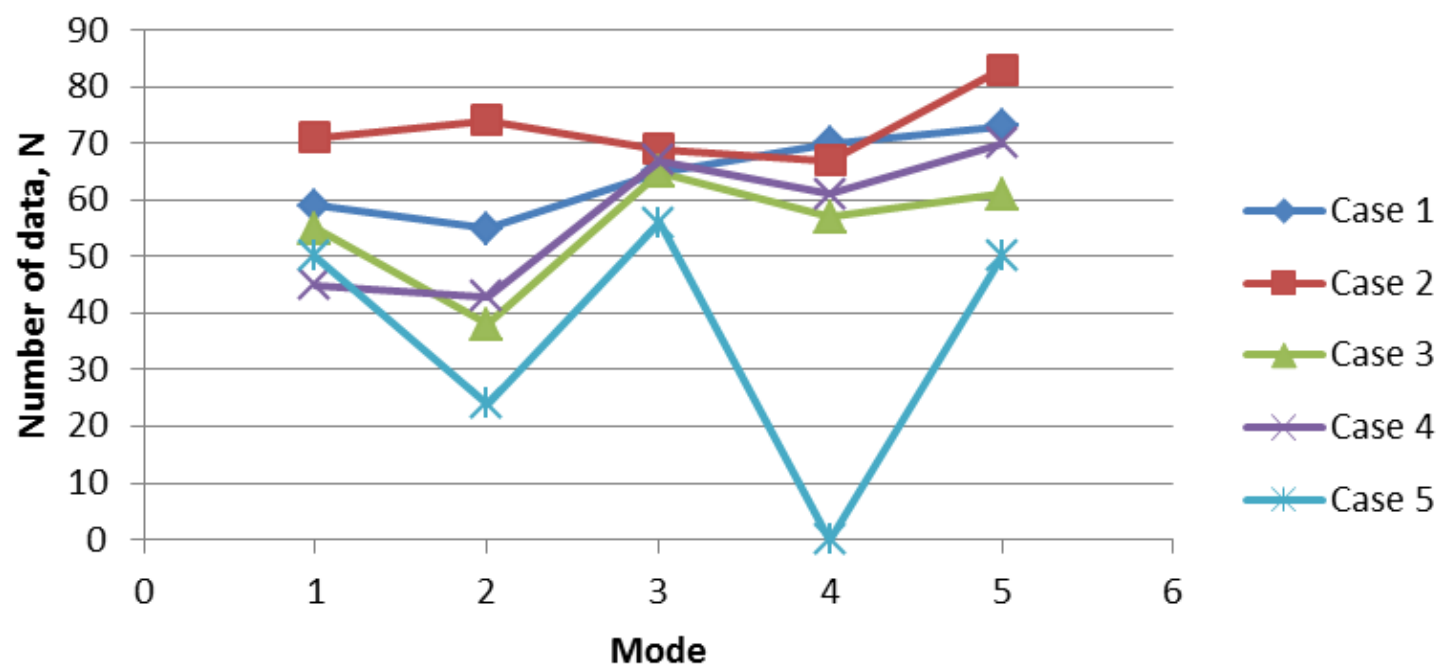

(f)

Fig 7: Number of data for all cases (a) SNR 0.1 (b) SNR 1 Glancing through situations corrupted with noise starting from SNR 0.1 to SNR 100 drew a number of attentions. It can be seen how the noise give significant impact towards number of data of each cases especially with shifted time interval. An exception is for Case 3 as together with Case 1, they produced greatest number of data consistently for each mode for all situations. Comparing both cases, Case 1 have larger number of data than Case 3 almost all the time. Thus, this is an elucidation for why Case 1 had lower percentage errors than Case 3 for most of the time as discussed previously. This indirectly also unlock one of the reason behind the robustness of Case 1 and Case 3 in surviving acceptable percentage errors for both parameter although the situation is corrupted extremely with noise.

The factor 'number of data' may also explain the erroneous result experienced by Case 2 and Case 4 at Mode 3 and Mode 4. This is clearly seen due to insufficient number of data at those modes. However, the effect is more observable for damping ratio instead of natural frequency although both

1 (c) SNR 10 (d) SNR 30 (e) SNR 60 (f) SNR 100 gained same pattern of locations for the errors to occur. This factor also gave answer to the lack in results yielded by Case 5. Another important notification is that the statement does not valid for situations SNR 60. As a result, an alternative explanation is required to remedy the problem.

\subsection{Data Distributions}

\subsubsection{Introduction to Data Distributions Analysis}

As mentioned earlier, number of data can play as an indicator in assessing the accuracy of results but the factor is not the primary indicator for this purpose. For example in situation SNR 60, Case 2 has sufficient number of data for all modes where the numbers surpassed the quantity of data for Case 1 except for Mode 4. At a glance, the majority of good results normally possessed approximately 50 data or higher referring only to Case 1 and Case 3 for all situations. These cases were selected as reference due to their consistencies in providing lower percentage error for each mode. With this definition, the difference in quantity for 
Mode 4 is considered small as Case 1 obtained 95 data while Case 2 with 82 data. However, Fig. 4 and Fig. 6 show that the percentage errors for damping ratio at Mode 3 and Mode 4 are $72.3 \%$ and $53.8 \%$ respectively whereas for Case 1 , the results are $0.009 \%$ and $0.003 \%$. The huge difference demands an alternative elucidation in fulfilling the missing understanding to this problem.

Other way of looking at this is by inspecting the pattern of data distributions and their closeness to the theoretical value. For this purpose, the following analysis would concentrate on situation SNR 60. The parameter investigated for this analysis only focusing on damping ratio as the impact of data quantity has less significance towards natural frequency. The term 'closeness' to the theoretical value will use the definition of acceptable percentage error where maximum deviation allowed from true values is \pm 0.05 . It is essential to not be confused between these two distinct terms as the limit for the percentage errors is related to the mean damping ratio while the term 'closeness' applied to all data that will contribute to the final value of mean damping ratio. Data residing outside this definition will be labelled as 'outliers'.

\subsubsection{Analysis of Data Distribution Results}

The following table listed the sample standard deviation and the mean at each mode for all cases for SNR 60:

Table 4: List of sample standard deviations for each mode and all case for SNR 60

\begin{tabular}{|l|l|l|l|l|l|}
\hline \multirow{2}{*}{$\begin{array}{l}\text { Mo } \\
\text { de }\end{array}$} & \multicolumn{5}{|l}{ Sample Standard Deviation } \\
\cline { 2 - 6 } & Case 1 & Case 2 & Case 3 & Case 4 & Case 5 \\
\hline 1 & 9.65645 & 0.00647 & 0.00326 & 0.04642 & 0.23585 \\
& E-06 & 871 & 8874 & 859 & 5401 \\
\hline 2 & $1.1991 \mathrm{E}$ & 0.00434 & 0.00115 & 0.00608 & 0.36542 \\
& -05 & 3426 & 2304 & 9391 & 1001 \\
\hline 3 & 3.47831 & 0.16454 & 0.00076 & 0.24799 & 0.11000 \\
& E-06 & 0102 & 094 & 8069 & 5963 \\
\hline 4 & 5.41418 & 0.08151 & 3.89237 & 0.13870 & 0.12389 \\
& E-06 & 4118 & E-05 & 4117 & 807 \\
\hline 5 & 0.00867 & 0.00317 & 0.01124 & 0.00883 & 0.02930 \\
& 5979 & 5876 & 0715 & 3524 & 2872 \\
\hline
\end{tabular}

Table 5: List of sample mean for each mode and all case for SNR 60

\begin{tabular}{|l|l|l|l|l|l|}
\hline \multirow{2}{*}{$\begin{array}{l}\text { Mo } \\
\text { de }\end{array}$} & \multicolumn{5}{|l}{ Sample Mean } \\
\cline { 2 - 6 } & Case 1 & Case 2 & Case 3 & Case 4 & Case 5 \\
\hline 1 & 0.29999 & 0.30090 & 0.29948 & 0.31530 & 0.26493 \\
& 8346 & 6747 & 6286 & 4064 & 8887 \\
\hline
\end{tabular}

\begin{tabular}{|l|l|l|l|l|l|}
\hline 2 & $\begin{array}{l}0.24999 \\
2192\end{array}$ & $\begin{array}{l}0.24921 \\
3822\end{array}$ & $\begin{array}{l}0.24965 \\
8943\end{array}$ & $\begin{array}{l}0.24826 \\
8181\end{array}$ & $\begin{array}{l}- \\
2.50940 \\
2018\end{array}$ \\
\hline 3 & 0.13889 & 0.03781 & 0.13900 & - & 0.07430 \\
& 016 & 1737 & 2891 & $\begin{array}{l}0.19160 \\
8597\end{array}$ & \\
\hline 4 & 0.09259 & 0.04281 & 0.09260 & - & - \\
& 4922 & 2912 & 0173 & $\begin{array}{l}0.09159 \\
4876\end{array}$ & $\begin{array}{l}0.68868 \\
5268\end{array}$ \\
\hline 5 & 0.04632 & 0.04669 & 0.04543 & 0.04507 & 0.05029 \\
& 0023 & 0393 & 6313 & 0485 & 8969 \\
\hline
\end{tabular}

From the Fig. 8, Table 4 and Table 5, the results depict that broad dispersion of data at certain mode lead to erroneous result for damping ratio. Case 2 as well as Case 4 at Mode 3 and Mode 4 exhibited that wide distribution of data influenced the average value of damping ratio. Monitoring closely at Fig. 8-b for Case 2 indicated that around 50 data were closed to the theoretical value, 0.139. However, with the presence of a number of outliers had affected the final average value to 0.038 . Similar pattern is also observed for Mode 4. These evidences showed that as the dissemination of data is wider, the more it affects the value for mean damping ratio which will also give impact towards percentage errors. This explains why Case 2 has high percentage errors for both modes although the quantities of data are 89 and 82 respectively.

It can also be seen that the dispersion of data for Case 4 is larger than Case 2. For Mode 3 and 4, Table 4 marked 0.165 and 0.082 for Case 2 whereas 0.248 and 0.139 for Case 4 . The vast spread of data for Case 4 at both modes had also influence the value for mean damping ratio as well as percentage error as shown in Fig. $8-\mathrm{g}$,h. Thus, the above statement is also valid for Case 4.

Switching to Case 5, the over-exceeding errors mostly occurred at Mode 2 and Mode 4. It is stated beforehand that the cause for this is due to insufficient number of data. The dissemination pattern for this condition illustrates that most of these points are very far from theoretical values by range of 0.6 to 3.5 thus affecting the mean damping ratio. The analysis using data distribution for Case 5 is not relevance as not much information can be extracted from this amount of quantity. 


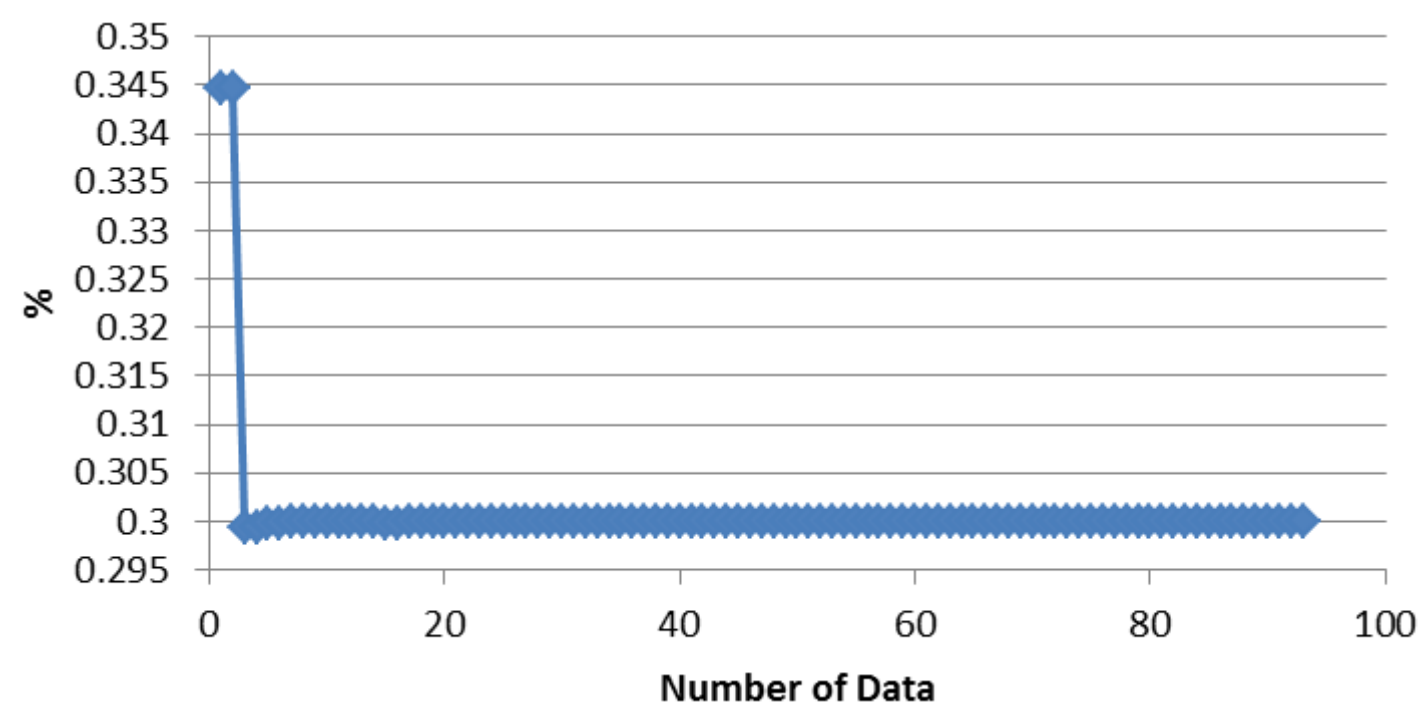

(a)

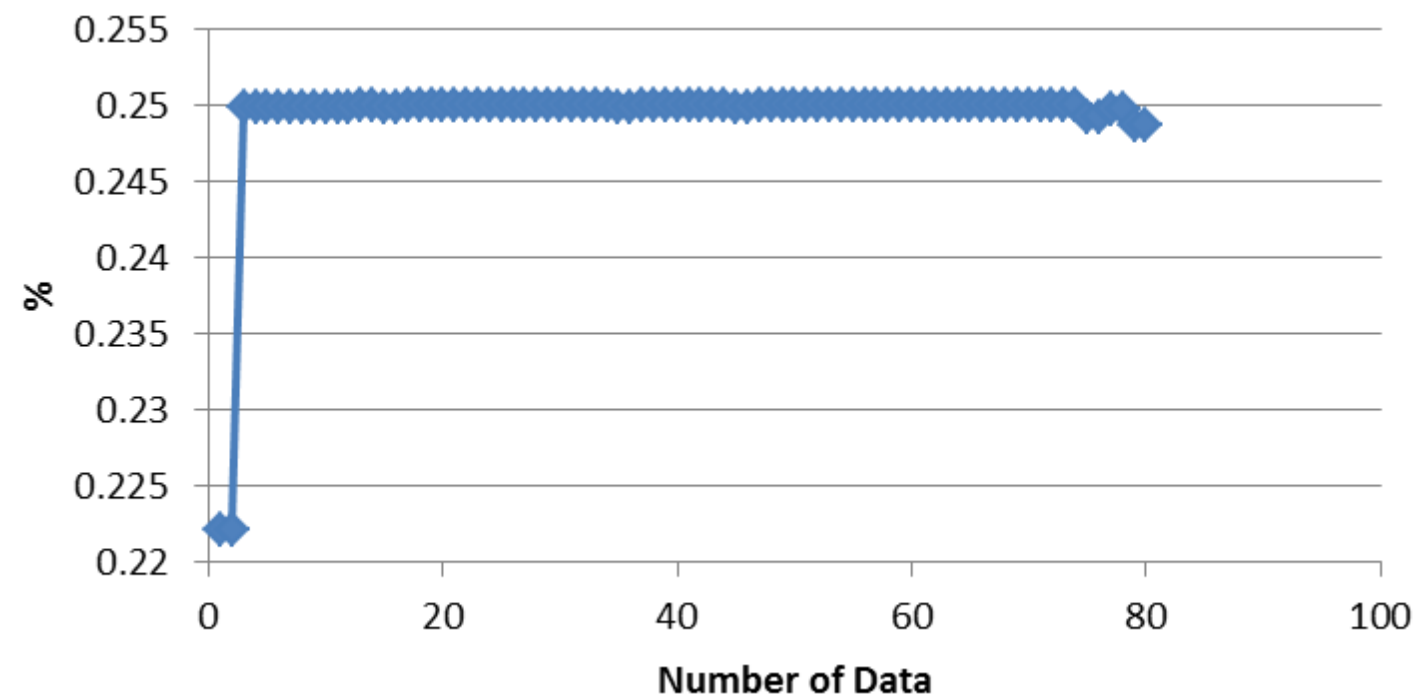

(b)

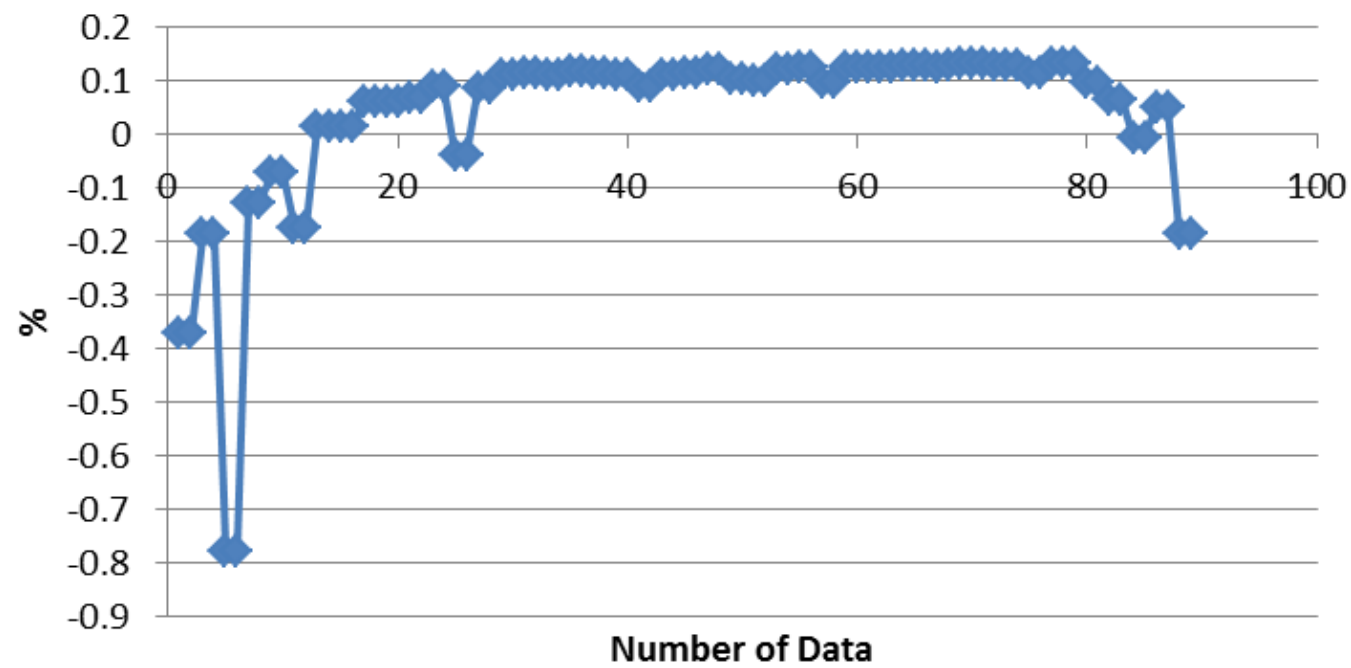

(c) 


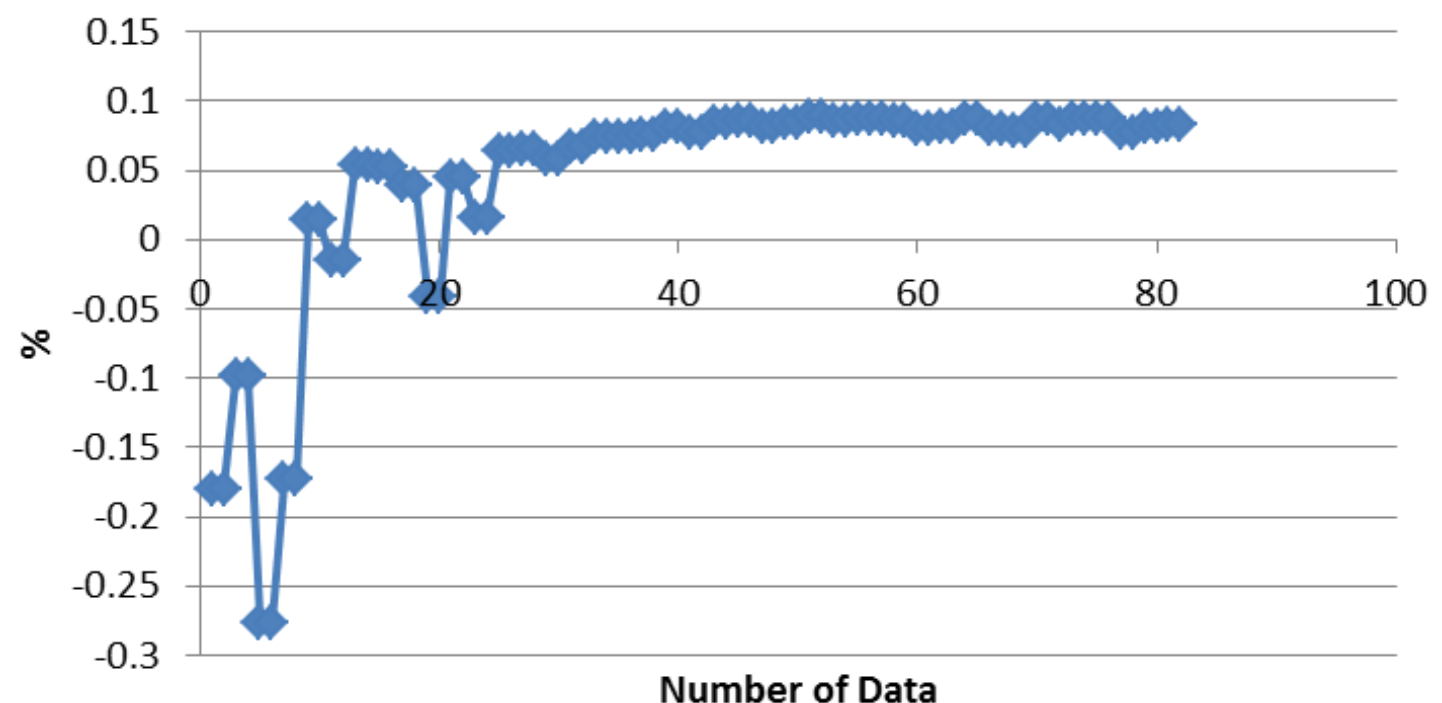

(d)

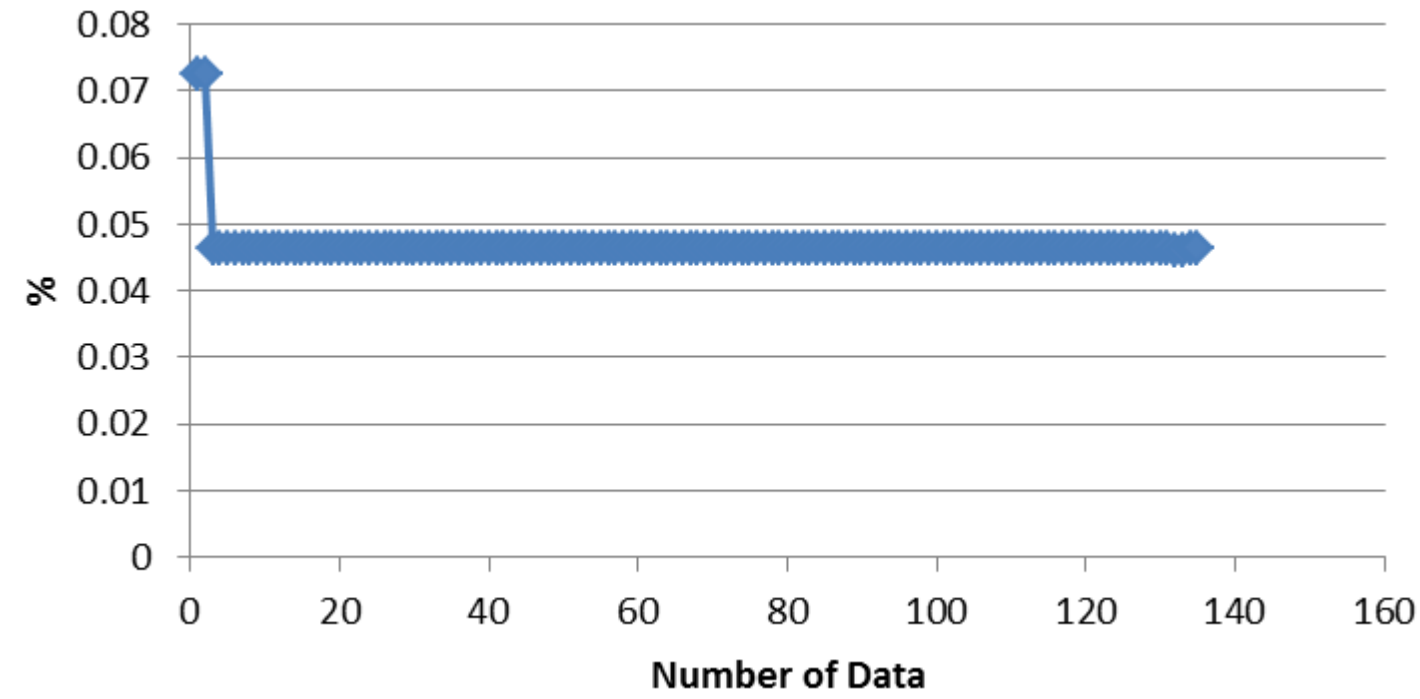

(e)

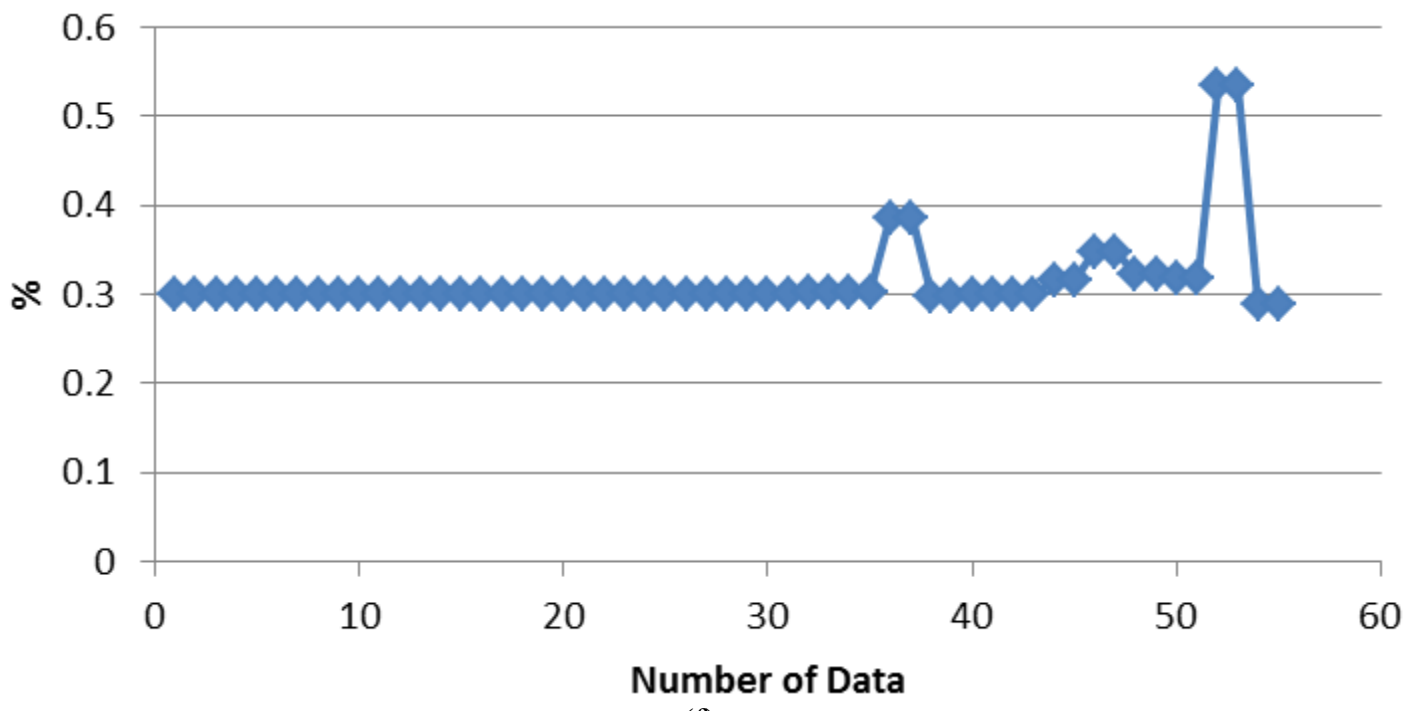

(f) 


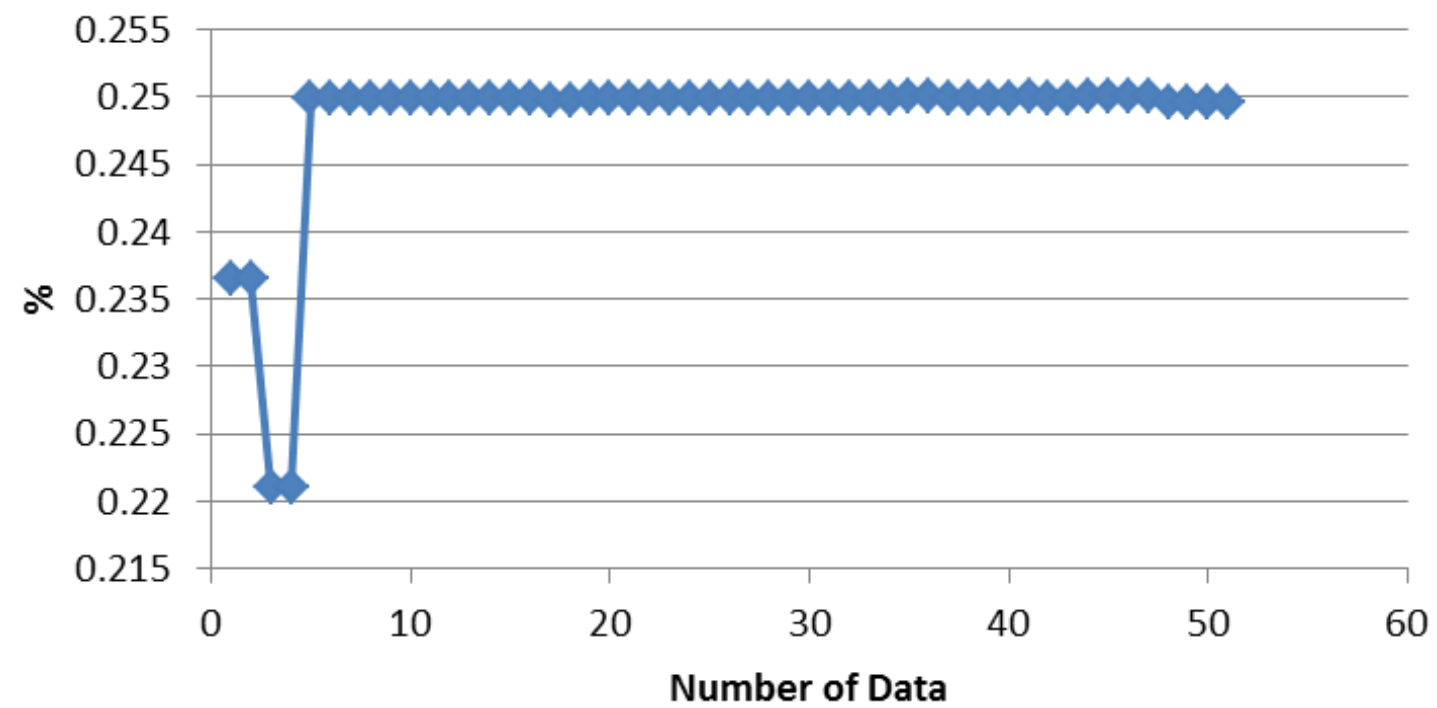

$(\mathrm{g})$

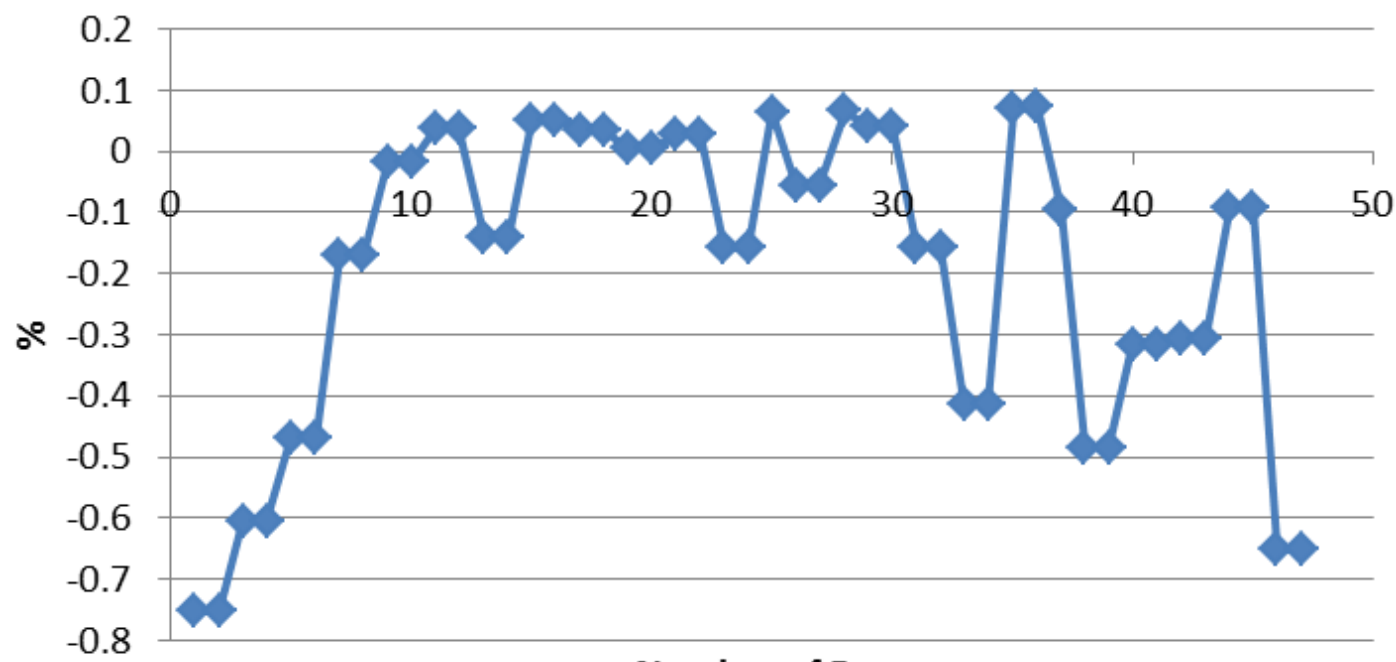

Number of Data

(h)

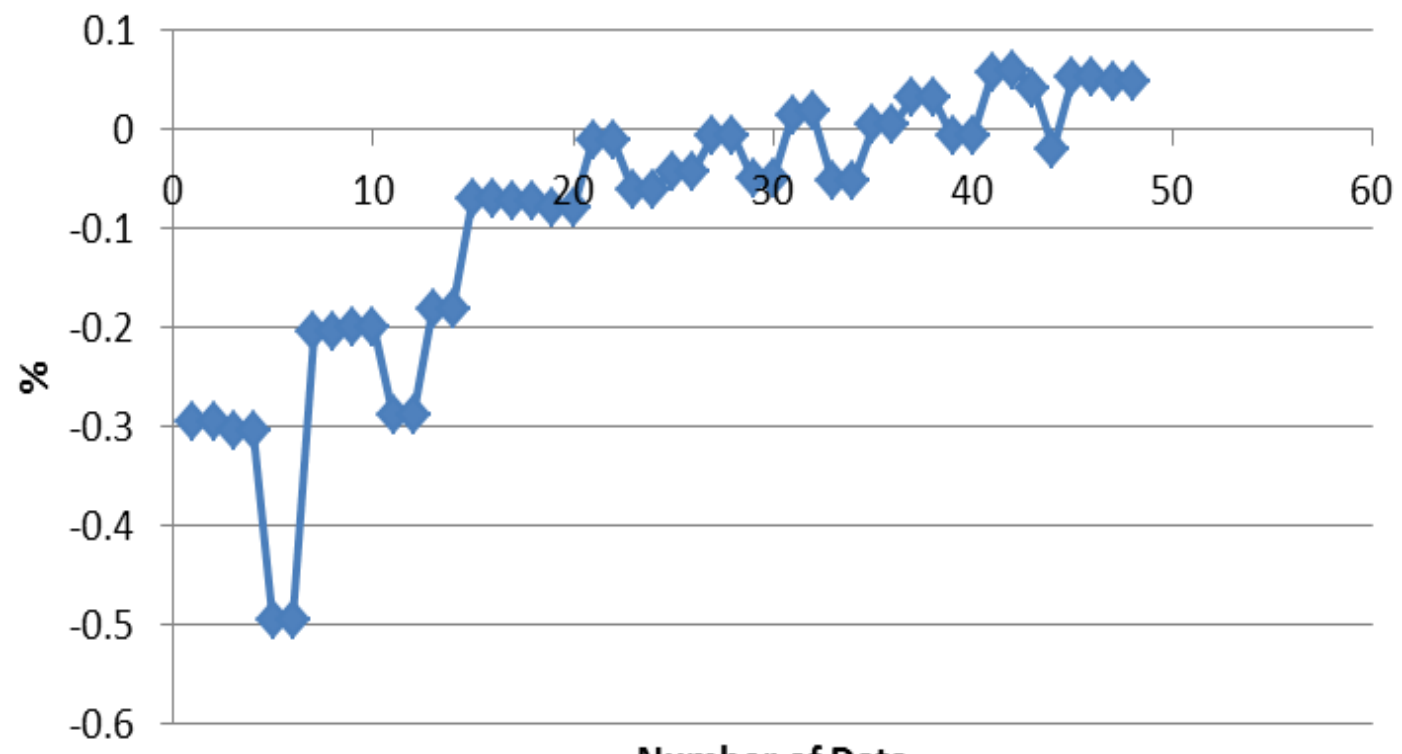

Number of Data

(i) 
Fig 8: Data distribution for Case 2 and Case 4, SNR 60 (a) Mode 1 Case 2 (b) Mode 2 Case 2 (c) Mode 3 Case 2 (d) Mode 4 Case 2 (e) Mode 5 Case 2 (f) Mode 1 Case 4 (g) Mode 2 Case 4 (h) Mode 3 Case 4 (i) Mode 4 Case 4 (j) Mode 5 Case 4

In comparison to Case 1 and Case 3, the distributions for all data at each mode are closed to each other and the theoretical value. Thus, there are no outliers as they meet the qualification of definitions although looking at the Graph seemed to illustrate some outliers.

An important note to realize is the source of these outliers. The primary role of MCF is to initially differentiate between structural and computational mode where in this study, value greater than 0.9 is chosen. The role is only effectively success for the default settings, Case 1 and also Case 3 where almost no outliers identified. For the rest of the cases, the quantity of these outliers is increased starting from Case 2 then Case 4. A number of deductions can be outlined from these phenomena:

1) As the shift in time interval is increased, the number of computational mode/outliers with value of MCF greater than 0.9 is also increased along with decreasing quantity of selected data and this will occur at certain mode. The presence of these outliers will influence the mean damping ratio and thus affecting the percentage errors. An exception is for Case 3 as no outliers identified in Graph. However, there is compromise between these two factors; number of outliers and quantity of data. A solid example is Case 5 where it has greatest reduction in number of data among all cases as obviously seen for Mode 2 and Mode 4 but these data are all outliers.

2) As the shift in time interval is increased, the error in value for computational mode/outliers that occur at certain mode is also increased. Quoting example of Case 5 again, the values of these outliers were very erroneous in comparison to the values of outliers from Case 4. This is also the same as the error in outliers for Case 4 is higher than Case 2.
3) As stated beforehand that in general, numbers of data can act as an indicator in assessing the accuracy of results but the factor is not the primary indicator for this purpose. This is due to that the factor is connected to other factor which is the amount of computational modes/outliers exist depending on how much shift in time interval for that case. More vitally, if all the data are all considered within the 'closeness' limit and have minimum 50 data but still having high percentage error, the cause rely on the pattern of dispersion among the data or from theoretical value if known. However, logically this should not happen as the 'closeness' limits introduced should ensure that the percentage error is in line with it.

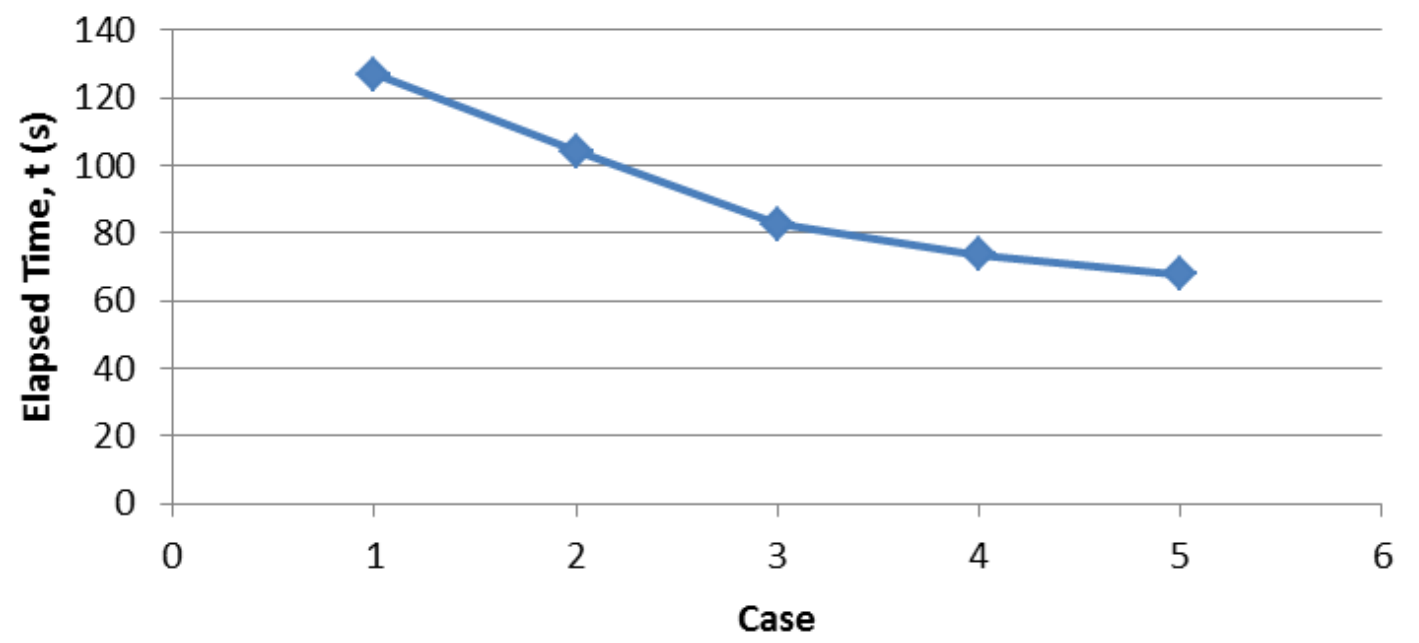

(a) 


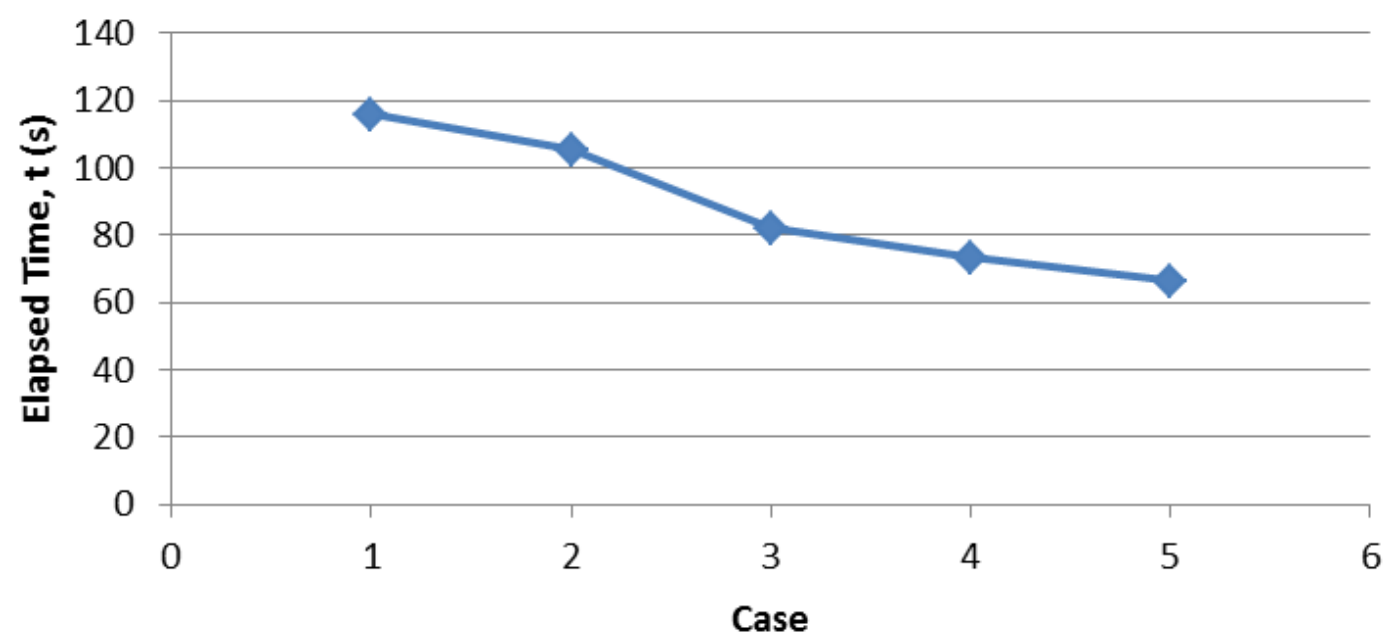

(b)

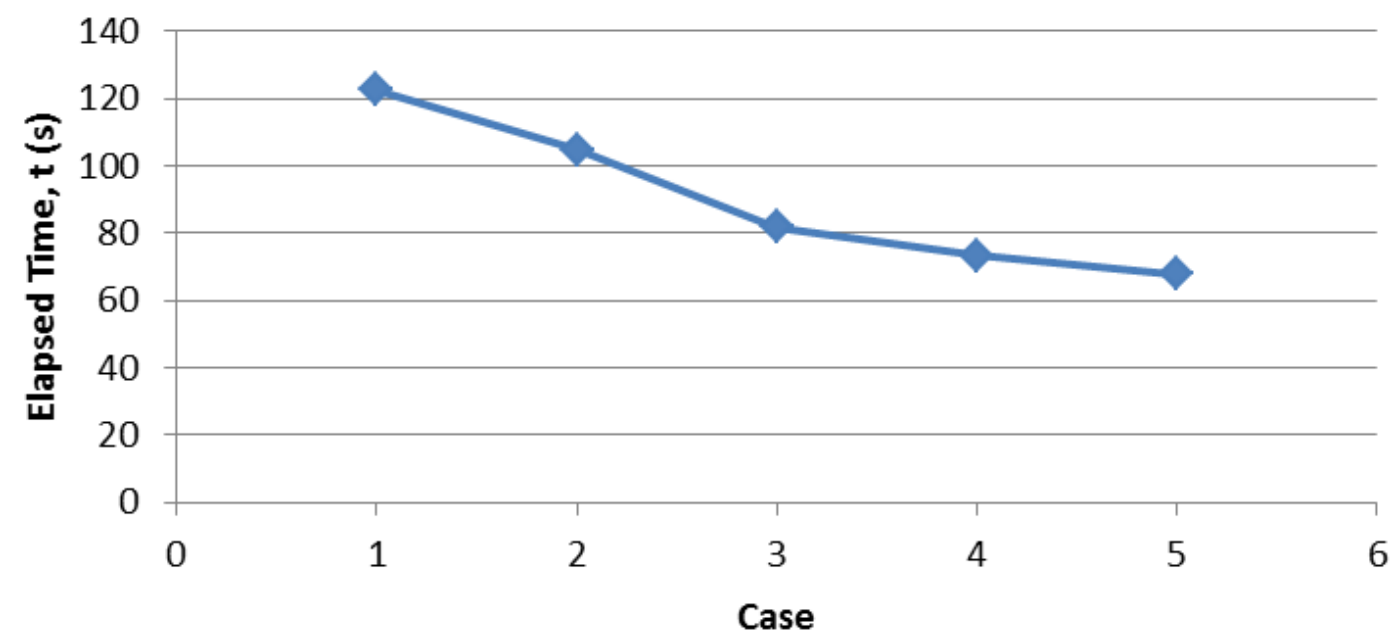

(c)

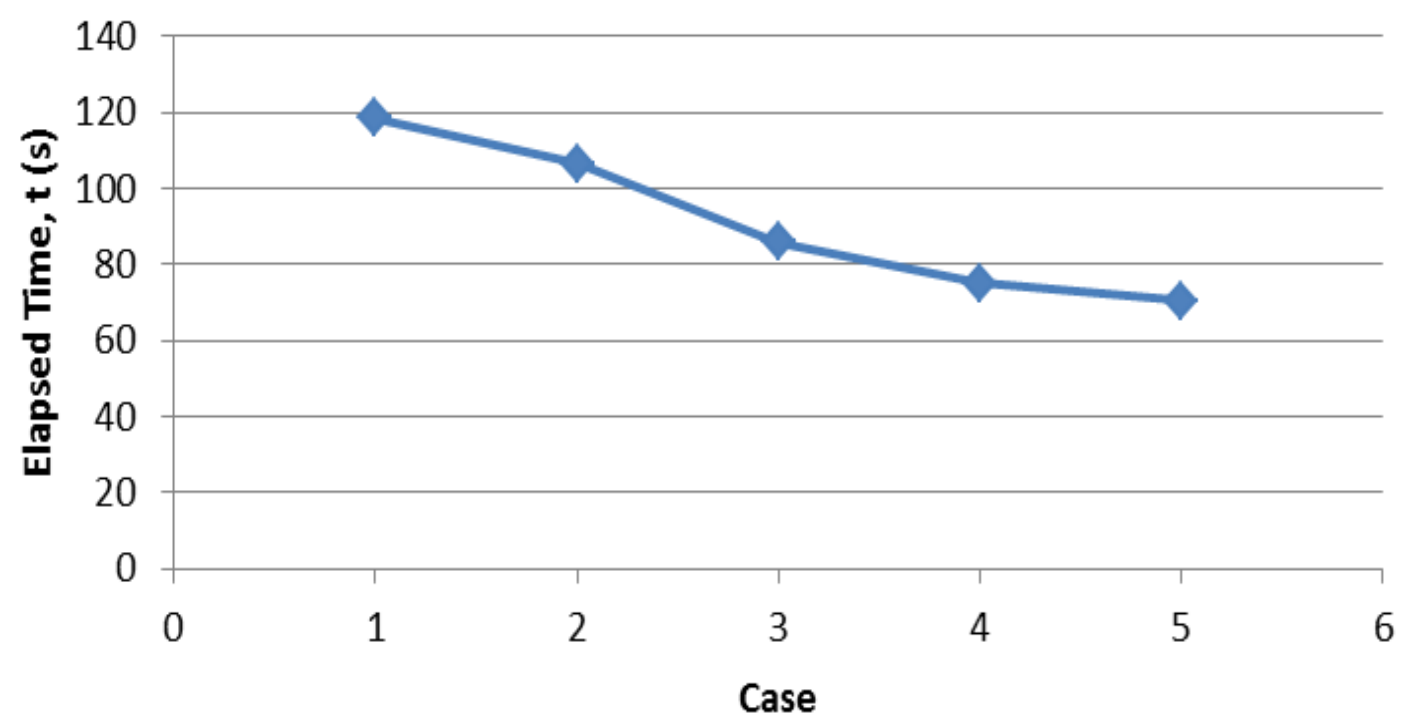

(d) 


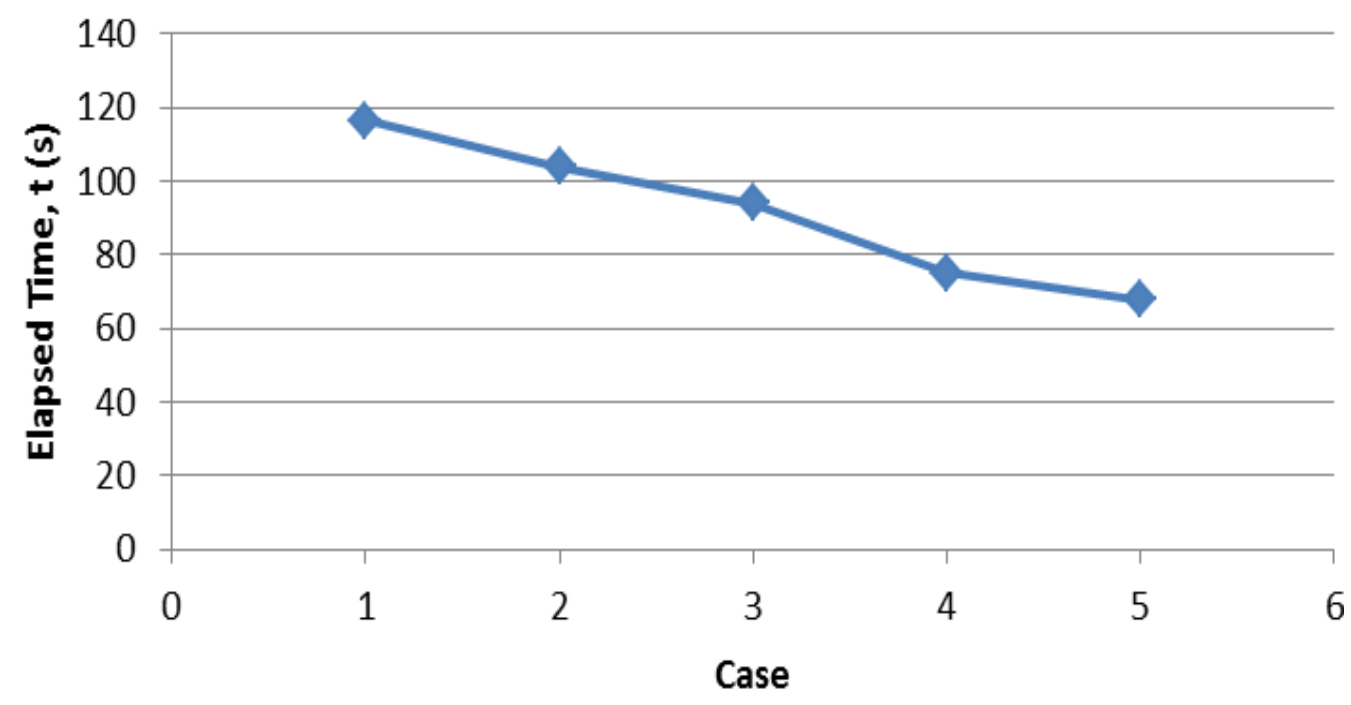

(e)

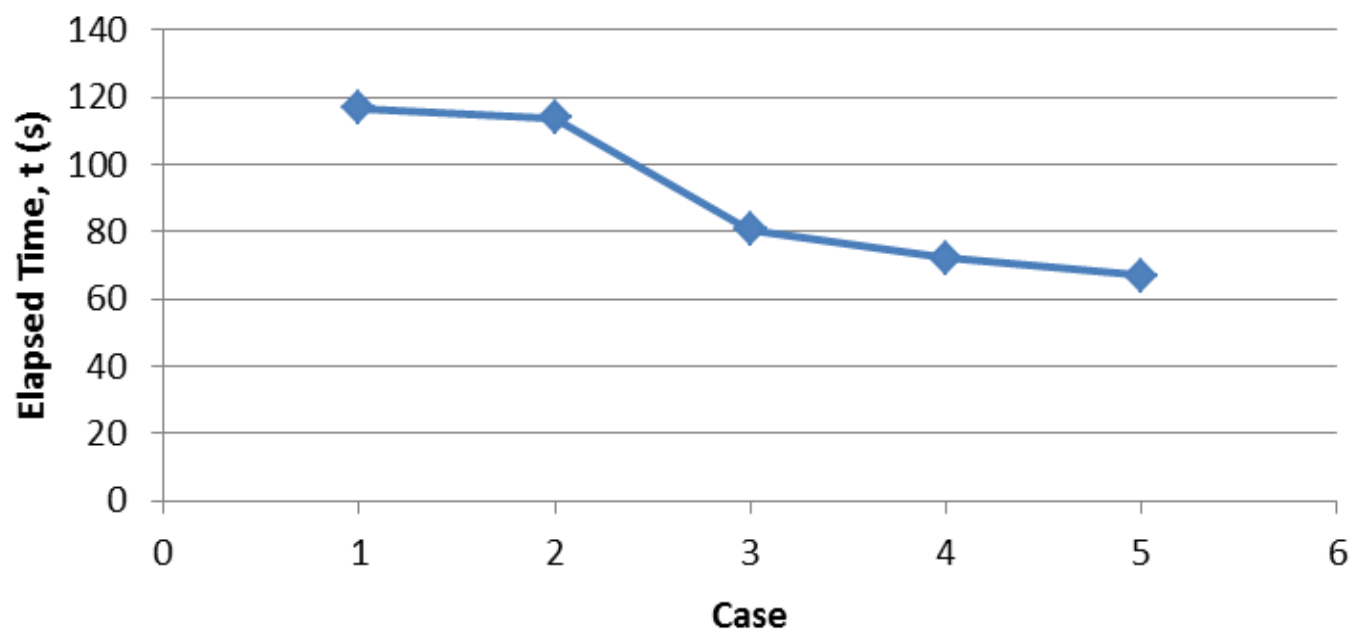

(f)

Fig 9: Time elapsed for all cases in each situation (a) SNR 0.1 (b) SNR 1 (c) SNR 10 (d) SNR 30 (e) SNR 60 (f) SNR 100

From these deductions, it is understandable that the reason affecting the level of broadness for data spread relies on the quantity and the values of these outliers that exist in the data. This give an answer on why at Mode 4, Case 1 has better results than Case 2 although both cases have the largest amount of data for that mode relative to other cases with 95 and 82 data respectively.

\subsection{Time Elapsed}

Fig. 9 exhibited the time taken for each case to calculate all the modal parameters. It can be seen that the time taken is proportional to the size of shift in time interval in general. In other words, this indicates that Case 1 has the longest time taken followed by Case 2, Case 3, Case 4 and finally Case 5 . The reduction in time for Case 5 is almost double from the default settings, Case 1. Thus, Case 3 can be used as alternative for large application of modal analysis project if time saving is the main concern as it also robust with slightly higher percentage error than Case 1.

\section{CONCLUSION}

In this paper, a numerical parametric study regarding the effect of time shift interval in SSTD algorithm for EMA had been carried out successfully. The results have shown that the default setting in SSTD algorithm which is Case 1 is robust and produce accurate result among all cases although in situations with high presence of noise. Case 3 is also robust as Case 1 but slightly less accurate. Following list stress out a number of vital conclusions deduced from the overall research work:

1) As the shift in time interval is increased, the misleading value for computational mode/outliers that occur at certain mode is also increased.

2) As the shift in time interval is increased, the quantity of computational mode/outliers with value of MCF greater than 0.9 is also increased in line with decreasing quantity of selected data. Results show that this will occur at certain mode only. 
3) The cause that influences the level of broadness for data spread relies on the quantity and the values of these outliers that exist in the data. Thus, it can also be stated that as dissemination of data is wider, the more it affects the value for mean damping ratio which will also give impact towards percentage errors.

4) In general, as the shift in time interval is increased, the number of data selected is decreased. This would lead to higher percentage error for both parameters.

5) The number of data is an indicator in assessing the accuracy of results but the quantity is not the sole indicator for this purpose.

6) Modes that have few numbers of data will yield poor results for damping ratio. However, this has less effect on natural frequency.

7) In general, as the amount of noise contained in the data is increased, the accuracy for natural frequency and damping ratio as well as quantity of data selected are decreased. For same amount of noise, the statement is true as the shift in time interval is increased. An exception is only for Case 3 where the results are robust even for high presence of noise.

\section{ACKNOWLEDGEMENTS}

The authors acknowledge with gratitude the great support from the management of Research Management Institute (RMI), UiTM amd financial support from the Minister of higher Education Malaysia (MOHE) for the granted grant of Fundamental Research Grant (FRGS).

\section{REFERENCES}

[1] Doebling, S.W., C.R. Farrar, and M.B. Prime, A summary review of vibration-based damage identification techniques. Shock and Vibration Digest, 1999. 205: p. 631-645

[2] Zhang, B.Q., G.P. Chen, and Q. Guo, Finite element model updating based on complex modal analysis for unsymmetrical damping system, in Computer Engineering and Technology (ICCET), 2010 2nd International Conference. 2010: Chengdu. p. V5-56 - V5-60

[3] Maia, N.M.M. and J.M.M.e. Silva, Theoritical and Experimental Modal Analysis. 1997: Research Studies Press, Cambridge, UK.

[4] Ewins, D.J., Modal Testing, Theory, Practise and Application. Second ed, ed. P.J.B. Roberts. 2000, London: Research Studies Press Ltd. 562

[5] He, J. and Zhi-Fang, Modal Analysis. 2001, Oxford, United Kingdom: Butterworth Heinemen. 291

[6] Maia, N.M.M. and J.M.M. Silva, Modal analysis identification techniques. Philosophical Transactions of the Royal Society A: Mathematical, Physical and Engineering Sciences, 2001. 359(1778): p. 29-40

[7] Cooley, J.W. and J.W. Tukey, An Algorithm for the Machine Calculation of Complex Fourier Series. Mathematics of Computation, 1965. 19(90): p. 297301

[8] Richardson, M. and D. Formenti, Parameter estimation from frequency response measurements using rational fraction polynomials, in Proceedings of the 1st International Modal Analysis Conference (IMAC I) Conference. 1982: Orlando, Florida, USA. p. 167-182

[9] Richardson, M. and D. Formenti, Global Curve Fitting of Frequency Response Measurements using the Rational Fraction Polynomial Method, in Proceedings of the 3rd International Modal Analysis Conference (IMAC III). 1985: Orlando, Florida, USA. p. 390-397

[10] Richardson, M.H., Global frequency and damping estimates from frequency response measurements, in Proceedings of the 4th International Modal Analysis Conference (IMAC IV). 1986: Los Angeles, California, U.S.A. p. 465-470

[11] Richardson, M. and D. Formenti, Parameter estimation from frequency response measurements using rational fraction polynomials (Twenty years of progress), in 2002 IMAC-XX: Conference \& Exposition on Structural Dynamics. 2002: Los Angeles, California, USA. p. 373-382

[12] Ewins, D.J. and P.T. Gleeson, A method for modal identification of lightly damped structures. Journal of Sound and Vibration, 1982. 84(1): p. 57-79.

[13] Avitabile, P., Experimental Modal Analysis, A Simple Non-Mathematical Presentation. Sound and Vibration, 2001. 35(Part 1): p. 20-31

[14] Alvin, K.F., et al., Structural system identification: from reality to models. Computers \& Structures, 2003. 81(12): p. 1149-1176

[15] Verboven, P., et al., Modal parameter estimation from input-output Fourier data using frequencydomain maximum likelihood identification. Journal of Sound and Vibration, 2004. 276(3-5): p. 957-979

[16] Ibrahim, S.R., Time Domain Modal Parameter Identification and Modeling of Structures, in American Control Conference. 1983. p. 989-996

[17] Ibrahim, S.R. and R.S. Pappa, Large modal survey testing using the Ibrahim Time Domain modal identification technique. Journal of Spacecraft and Rockets, 1981. 19(5): p. 459-465

[18] Ibrahim, S.R. and R.S. Pappa, A Parametric Study of The Ibrahim Time Domain Modal Identification Algorithm. Shock and Vibration Bulletin, 1981. 51(3): p. 43-73.

[19] Zaghlool, S.A., Single-Station Time-Domain (SSTD) Vibration Testing Technique: Theory and Application. Journal of Sound and Vibration, 1980. 72(2): p. 205-234.

[20] Mohanty, P., Modified SSTD method to account for harmonic excitations during operational modal analysis. Mechanism and Machine Theory, 2004. 39(12): p. 1247-1255

[21] Ibrahim, S.R., Double least square approach for use in structural modal identification. AIAA Journal, 1986. 24: p. 499-503

[22] Nayeri, R.D., et al., Study of Time-Domain Techniques for Modal Parameter Identification of a Long Suspension Bridge with Dense Sensor Arrays. Journal of Engineering Mechanics, 2009. 135(7): p. 669-683 
[23] Adhikari, S., Damping Models for Structural Vibration, in Engineering Department. 2000, University of Cambridge: Cambridge. p. 235

[24] Oliveto, G. and A. Greco, Some Observations on the Characterization of Structural Damping. Journal of Sound and Vibration, 2002. 256(3): p. 391-415

[25] Barkanov, E., W. Hufenbach, and L. Kroll, Transient response analysis of systems with different damping models. Computer Methods in Applied Mechanics and Engineering, 2003. 192(12): p. 33-46

[26] Li, X.Y. and S.S. Law, Identification of structural damping in time domain. Journal of Sound and Vibration, 2009. 328(1-2): p. 71-84

\section{BIOGRAPHIES}

Ahmad Fathi Ghazali is a postgraduate student at at Mechanical Engineering Faculty, MARA university of Technology, 40450 Shah Alam, Malaysia

Ahmad Azlan Mat Isa is a lecturer at Mechanical Engineering Faculty, MARA University of Technology, 40450 Shah Alam, Malaysia 